\title{
Figural properties are prioritized for search under conditions of uncertainty: Setting boundary conditions on claims that figures automatically attract attention
}

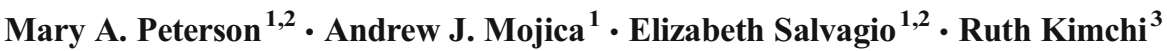

Published online: 28 October 2016

(C) The Psychonomic Society, Inc. 2016

\begin{abstract}
Nelson and Palmer (2007) concluded that figures/ figural properties automatically attract attention, after they found that participants were faster to detect/discriminate targets appearing where a portion of a familiar object was suggested in an otherwise ambiguous display. We investigated whether these effects are truly automatic and whether they generalize to another figural property - convexity. We found that Nelson and Palmer's results do generalize to convexity, but only when participants are uncertain regarding when and where the target will appear. Dependence on uncertainty regarding target location/timing was also observed for familiarity. Thus, although we could replicate and extend Nelson and Palmer's results, our experiments showed that figures do not automatically draw attention. In addition, our research went beyond Nelson and Palmer's, in that we were able to separate figural properties from perceived figures. Because figural properties are regularities that predict where objects lie in the visual field, our results join other evidence that regularities in the environment can attract attention. More generally, our results are consistent with Bayesian theories in which priors are given more weight under conditions of uncertainty.
\end{abstract}

Keywords Selective attention · Grouping · Figure-ground segregtation $\cdot$ Perceptual organization

Mary A. Peterson

mapeters@u.arizona.edu

1 Psychology Department, University of Arizona, Tucson, Arizona, USA

2 Cognitive Science Program, University of Arizona, Tucson, Arizona, USA

3 Psychology Department, University of Haifa, Haifa, Israel
Questions concerning the relationship between objects and attention have long fascinated scientists. One recurring question is whether objects have a special status with respect to the allocation of attention. For instance, there is evidence that once attention is allocated to one part of an object (say, by a precue), observers attend to other locations on the same object more efficiently (faster/more accurately) than to equidistant locations on other objects (Egly, Driver, \& Rafal, 1994). This behavior, termed object-based attention, was long thought to be automatic (e.g., Richard, Lee, \& Vecera, 2008; Yeari \& Goldsmith, 2010). But in a series of elegant studies, Shomstein and Yantis $(2002,2004)$ showed that object-based attention is not automatic; it is observed only when participants are uncertain regarding where a target will appear. Under these conditions, it is expedient to begin searching for the target on the cued object.

A related question is whether, without a precue, attention is automatically drawn to objects. Kimchi and colleagues (Kimchi, Yeshurun, \& Cohen-Savransky, 2007; Kimchi, Yeshurun, Spehar, \& Pirkner, 2016; Yeshurun, Kimchi, Sha'shoua, \& Carmel, 2009) have demonstrated that a perceptual object (elements in the visual scene organized by Gestalt factors into a coherent unit) can capture attention. Of particular relevance to the present article is whether - again without a precue-attention is automatically drawn to the insides of objects, or figures, as compared to the regions immediately outside their borders. A critical feature of figures is that they are perceived as shaped by the border they share with abutting regions, whereas the abutting regions, lacking a contour, appear locally shapeless and seem to continue behind the figure. Some research has shown that when there are sufficient depth cues to indicate which of two surfaces is closer to the viewer, the closer one receives processing priority (e.g., Lester, Hecht, $\&$ Vecera, 2009). This effect seems to be automatic, since it is obtained even when the target and display onset 
simultaneously (West, Pratt, \& Peterson, 2013). Those findings do not entail that figures automatically attract attention, however, because the depth step between a figure and its (back)ground can be quite small. Hence, the question of whether figures automatically attract attention remains unanswered.

Here we investigated whether attention is automatically allocated to the side of a border that is perceived to have shape (the figure side), rather than to the adjacent ground side. Determining whether attention is automatically allocated to the figural side of a border (or the side where figural priors lie) rather than to the adjacent ground side is important to understanding how perceptual organization and attention interact (cf. Robertson \& Kim, 1999; Wager, Peterson, Folstein, $\&$ Scalf, 2015), yet surprisingly little research has been directed to answering this question. Hochberg (1971) suggested that attention is more often allocated to figures than to grounds, but this claim entails habitual rather than automatic behaviors. Using the Rubin vase-faces stimulus, Wong and Weisstein (1982) showed enhanced orientation discrimination for targets presented on a given region (e.g., the center black region or the surrounding white regions in Fig. 1) when participants reported they were perceiving that region as the figure rather than the other region. Wong and Weisstein's design, however, may have led participants to attend to the region they were perceiving as figure to monitor whether they were maintaining that percept; thus, Wong and Weisstein's findings may have reflected strategic rather than automatic attentional allocation (cf. Nelson \& Palmer, 2007; Peterson \& Gibson, 1993).

More recently, Nelson and Palmer (2007) directly addressed the question of whether figures attract attention. They used vertically elongated rectangular displays divided in half (bipartite displays) by a contour that suggested a portion of a familiar object on one side (Fig. 2). Familiar configuration is an object property that predicts, with high probability, where a figure lies with respect to a border (Peterson \& Gibson, 1994a, 1994b; Peterson, Harvey, \& Weidenbacher,

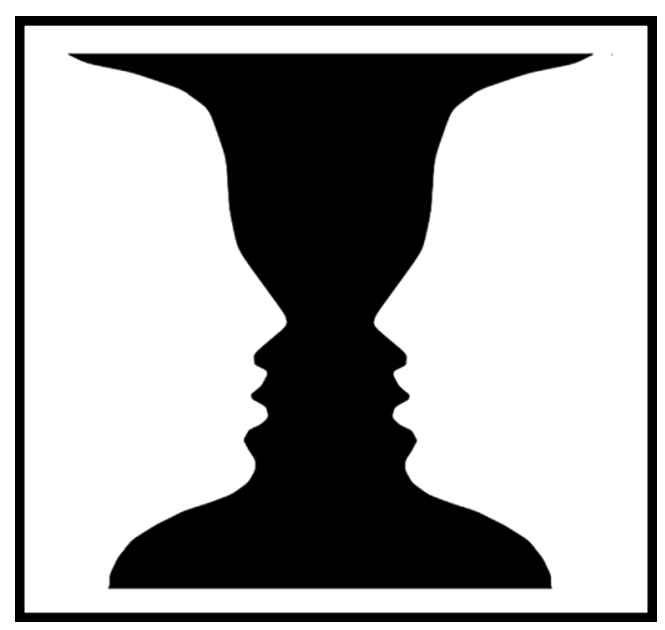

Fig. 1 The Rubin vase-faces stimulus

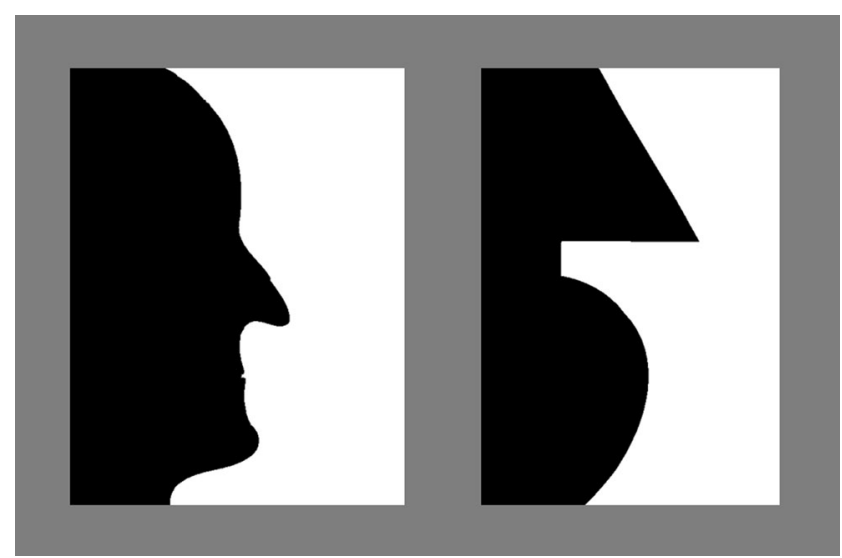

Fig. 2 Bipartite displays similar to those used by Nelson and Palmer (2007). Both displays depict a portion of a familiar object on one side of a central border, a face profile in black in the display on the left, and a table lamp in black in the display on the right. (Black/white fill and left/ right location were balanced in the experiment.) These displays were first published as Fig. 2 of "Object Recognition Contributions to FigureGround Organization: Operations on Outlines and Subjective Contours," by Peterson and Gibson 1994a, Perception \& Psychophysics, 56, page 554. Reprinted with permission of the Psychonomic Society

1991; for reviews, see Peterson, 1994; Peterson \& SkowGrant, 2003). Nelson and Palmer's participants performed a detection or discrimination task regarding targets that appeared equally often on both sides of the central border at various delays after display onset. The participants responded faster and more accurately to targets presented on the familiar configuration side of the border rather than on the opposite, complementary, side. Since responses are typically faster to targets presented in attended rather than unattended locations (e.g., Jonides, 1981; Yantis \& Jonides, 1984), these results led Nelson and Palmer to conclude, "the visual system processes information from the figural region more quickly than information from the ground region" (p. 385). They argued further that the figural advantage is automatic, reasoning that participants had no incentive to adopt a strategy of attending to the familiar configuration side of the display, given that the targets appeared equally often on both sides of the display, and doing so could not benefit performance. Nelson and Palmer also raised the intriguing possibility that perhaps figural properties - those that predict where figures are located with respect to a border - attract attention, rather than the perceived figures per se. They did not attempt to separate the role of figural properties from that of perceived figural status, however. Indeed, it would have been difficult to do so, because the figural property they manipulated - familiar configurationis highly likely to determine where the figure lies with respect to the central border of two-region displays (e.g., Gibson \& Peterson, 1994; Peterson, Gerhardstein, Mennemeier, \& Rapcsak, 1998). Consequently, the number of trials on which the complementary region was perceived as figure would have 
been too small to permit the separation of figural status and figural property.

\section{Précis}

In the experiments reported in this article, we investigated whether figures automatically attract attention. We began by testing whether Nelson and Palmer's (2007) effects generalize to another figural property-convexity. We found that they do, but only when participants are uncertain regarding when and where the target will appear, and must search for it. We found the same dependence on uncertainty for the figural property of familiarity originally tested by Nelson and Palmer. Thus, our results show that the allocation of attention to figures is not automatic, but instead occurs under conditions of uncertainty. Furthermore, because convex regions are less likely to be perceived as figures than are regions suggesting familiar configurations (Peterson \& Salvagio, 2008), our tests of convexity allowed us to assay whether attention is attracted to perceived figures or to the figural properties that predict where figures are likely to lie. Our results showed that under conditions of uncertainty, attention is directed to figural properties rather than to figures per se. Given that figural properties are priors for figures (Goldreich \& Peterson, 2012), our results extend the previous evidence that priors are given more weight under conditions of uncertainty (cf. Bisley \& Goldberg, 2010; Eckstein, 2011; Körding \& Wolpert, 2004; Shomstein, 2012).

\section{Experiment 1}

In Experiment 1, we explored whether we could extend Nelson and Palmer's (2007) findings that targets are detected faster when they appear on figures rather than on grounds, obtained when figures were defined by familiarity, to the figural property of convexity. We reasoned that if figures automatically attract attention, Nelson and Palmer's finding should generalize to convexity.

Ample evidence has revealed that convex regions are more likely to be perceived as figures than abutting, concave regions when displays comprise multiple convex regions alternating with multiple concave regions. For instance, observers reported perceiving the convex regions as figures on $90 \%$ of trials when they viewed displays like those in Fig. 3 (Kanizsa \& Gerbino, 1976; Peterson \& Salvagio, 2008). Peterson and Salvagio (2008; cf. Goldreich \& Peterson, 2012) showed that convex regions were substantially less likely to be perceived as figures in displays comprising two regions (one convex and one concave region, as in Fig. 7) than in displays comprising eight regions (four convex regions alternating with four concave regions, as in Fig. 3). Moreover, although convex regions

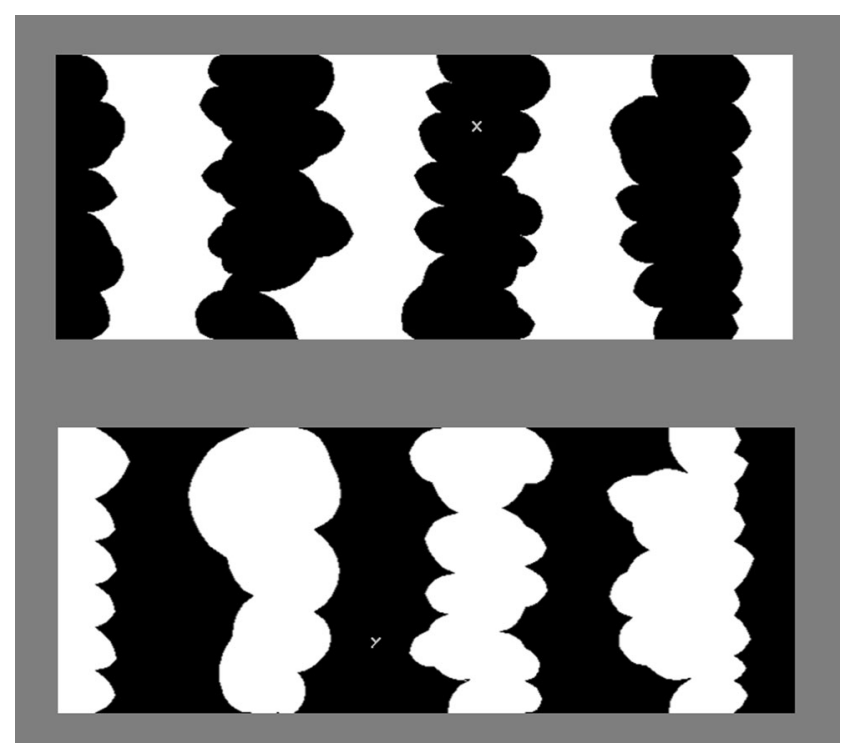

Fig. 3 Sample eight-region displays with alternating convex and concave regions used in Experiments 1A and 1B. The convex regions are black in the top display and white in the bottom display. Sample targets are shown, an " $x$ " on the convex region to the right of the central border in the top display, and a " $y$ " on the concave region to the left of the central border in the bottom display. Targets were red in the experiments, but are shown here in gray for visibility

were perceived as figures in two-region displays more often than would be expected by chance alone (on 57-60\% of trials), the effect of convexity on figure assignment was not large. Therefore, to conduct a sensitive test of whether convex figures attract attention, in Experiment 1 we began by using eight-region rather than two-region (bipartite) displays like those Nelson and Palmer (2007) had used.

In both Experiments 1A and 1B, eight-region displays appeared, centered on fixation. The target letters- " $x$ " and "y"-appeared at various stimulus onset asynchronies (SOAs) after the onset of the display, but equally often on the convex and on the concave side of the central border. Participants' task was to identify the target letter as an " $\mathrm{x}$ " or a "y." In Experiment 1A, the target was exposed until the participants responded. Here, accuracy was expected to be high; our dependent measure was reaction time (RT). In Experiment 1B, the target was exposed briefly and masked. Here, accuracy was expected to be low; our dependent measure was accuracy on a target discrimination task (transformed into a sensitivity measure: $d^{\prime}$ ).

These two experiments were modeled on those of Nelson and Palmer (2007), in which they found better discrimination of targets shown on figures than of those on grounds in terms of both RTs, with long exposures, and sensitivity $\left(d^{\prime}\right)$, with short exposures. In both Experiments $1 \mathrm{~A}$ and $1 \mathrm{~B}$, however, we failed to find evidence of better discrimination of targets shown on convex figures than of those on the abutting grounds. 


\section{Method}

\section{Participants}

A total of 24 (19 females, five males) undergraduate students from the University of Haifa participated in this study after giving informed consent; they took part in the experiment to partially fulfill the requirements for their introductory psychology class. Of these participants, 12 (ten females, two males) participated in Experiment $1 \mathrm{~A}$, and the remaining 12 (nine females, three males) participated in Experiment 1B. All participants reported normal or corrected-to-normal visual acuity.

\section{Stimuli and apparatus}

The stimuli were a subset of Peterson and Salvagio's (2008) eight-region displays, composed of black and white alternating convex and concave regions. These displays were presented within a virtual rectangular frame; this frame cut the left- and rightmost regions in half, creating the impression of partial occlusion. ${ }^{1}$ The convex and concave portions of these displays were equal in area. In half of the displays the region to the left of the central border was convex, and in the other half it was concave (see Peterson \& Salvagio, 2008, for details about the stimulus construction; to download the stimuli, see http://petersonlab.wix.com/visualperceptionlab\#!research/).

There were 48 unique black-and-white test displays (black: RGB 0, 0, 0; white: RGB 255, 255, 255). The black/white fill in the convex regions was balanced. All displays were equal in size, subtending a visual angle of $7.3^{\circ}$ high $(\mathrm{H})$ and $18.4^{\circ}$ wide (W). Each full region was approximately $2.3^{\circ}$ wide.

A black fixation cross $\left(0.50^{\circ} \mathrm{H} \times 0.50^{\circ} \mathrm{W}\right.$; RGB, $\left.0,0,0\right)$ was presented where the central border of the upcoming test display would be located. A red target letter $\left(0.27^{\circ} \mathrm{H} \times\right.$ $\left.0.27^{\circ} \mathrm{W} ; \mathrm{RGB}, 255,0,0\right)$ appeared approximately $1.8^{\circ}$ above or below the fixation location and was centered in a convex or concave region to the left or right of the central edge. The mask following the target presentation in Experiment 1B was $0.75^{\circ} \mathrm{H} \times 0.75^{\circ} \mathrm{W}$ and consisted of a small array of random black dots $(\mathrm{RGB}=0,0,0)$ on a white background (RGB 255, 255, 255). The test displays were centered on a screen that provided a medium gray backdrop.

A 17-in. CRT monitor controlled by a personal computer was used to present the stimuli and to record responses. Participants viewed the monitor from a viewing distance of $60 \mathrm{~cm}$, with distance constrained by a chinrest. Responses

\footnotetext{
${ }^{1}$ This is the standard method used by investigators to ensure that the convex and concave regions are equal in area and are truncated equally often. See Mojica and Peterson (2014) for evidence that the shape of the outer borders is critical.
}

were recorded via a custom button box with two horizontally arranged buttons. The visual stimulus presentation was controlled by code developed with the Delphi toolkit.

\section{Procedure}

Participants were instructed using computer-displayed instructions on the nature of their task. They were told that a display with multiple black and white regions would appear briefly, and after a variable delay, a target letter would appear on the left or right side of the central border. They were told that their task was to identify the target letter as either an " $x$ " or a " $y$ " by using their dominant hand to press either the left or the right button on a custom-designed button box (assignment of the right and left buttons to the " $x$ " and " $y$ " responses was balanced across participants).

Each trial began with a central fixation cross, appearing for $750 \mathrm{~ms}$. The fixation cross was followed by an eight-region black-and-white display. The two target letters were equally likely to appear and appeared equally often in one of the four locations (on the left or right side of the central border and above or below fixation) at one of four SOAs: $0,150,250$, or $500 \mathrm{~ms}$ after the onset of the display. Participants' task was to report the identity of the target letter. In Experiment 1A, the target remained visible until a response was received, for a maximum of 2,000 $\mathrm{ms}$ (a time-out was recorded in the latter case). In Experiment 1B, the target disappeared after $80 \mathrm{~ms}$ and was followed immediately by a mask. A sample trial is shown in Fig. 4.

Participants completed 640 experimental trials (in four blocks of 160 trials each), preceded by eight practice trials. None of the displays used on practice trials appeared during the experimental trials. An experimenter stayed in the room during the instructions and practice trials to answer any questions. Participants received feedback on all trials. If they made an incorrect response, an auditory tone was played, and no feedback was given for correct responses.

\section{Results}

\section{Experiment $1 A$}

Accuracy In Experiment 1A, in which the target remained visible until response, accuracy was high (mean $d^{\prime}=4.64$ ) and we observed no main effects of Region Type ${ }^{2}$ (convex or concave) or SOA $(0,150,250$, or $500 \mathrm{~ms})$, all $p \mathrm{~s}>.20$.

Reaction times In this and the subsequent experiments, we report analyses conducted on participants' RTs only on trials on which they accurately identified the target. We followed a

\footnotetext{
${ }^{2}$ We use the term "Region Type" for Experiment 1 because the stimuli included regions bounded on both sides by convex (or concave borders).
} 


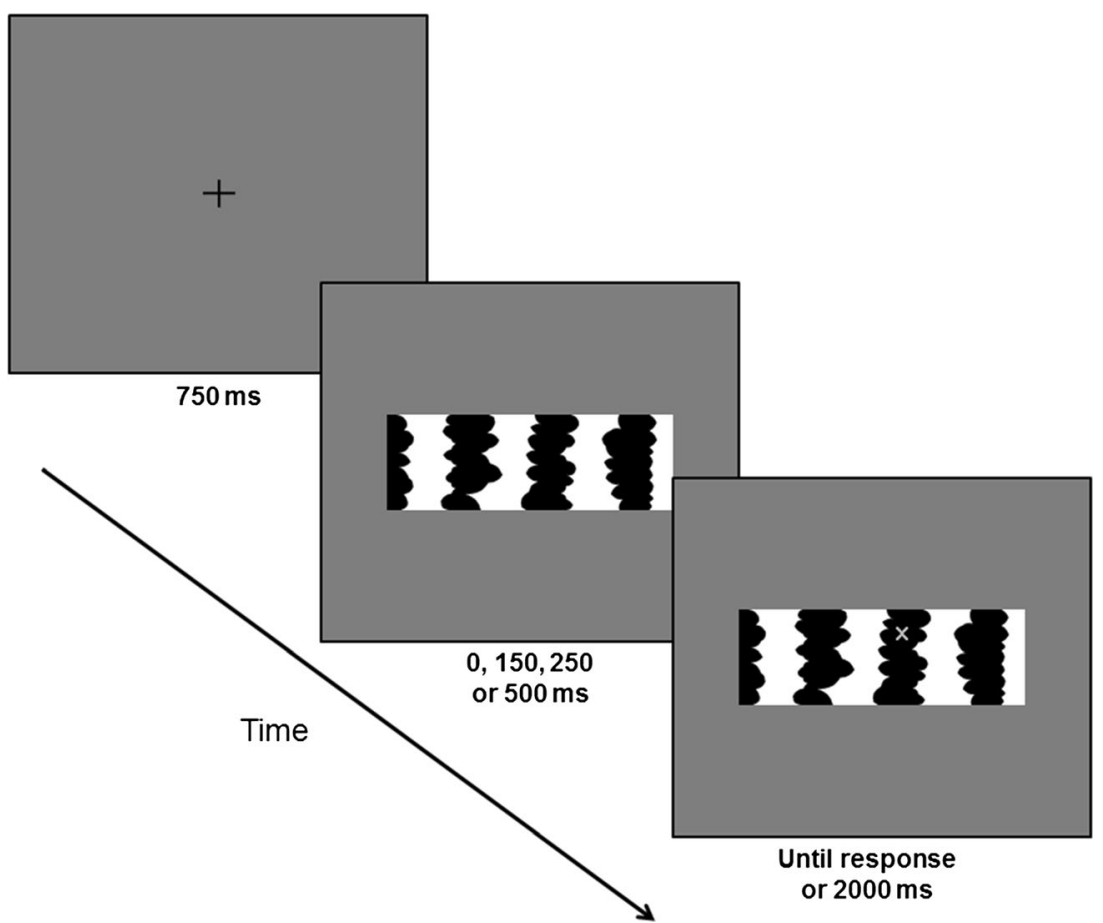

Fig. 4 Experiment 1A trial sequence. Target size is increased here for visibility

procedure of removing the data from participants whose RTs were more than two standard deviations from the condition mean. We conducted an analysis of variance (ANOVA) on RTs recorded on accurate trials, with two within-subjects factors: Region Type (convex vs. concave) and SOA (0, 150, 250 , or $500 \mathrm{~ms}$ ). We obtained a main effect of region type, $F(1,11)=37.59, p<.001$, but in the opposite direction from what we would expect on the basis of Nelson and Palmer's (2007) results: Participants' RTs were faster for targets located on the concave (ground) region $(652.3 \mathrm{~ms}$ ) than for those on the convex (figure) region (660.1 ms; see Fig. 5A). There was also a main effect of SOA, $F(3,33)=74.62, p<.001$, in which RTs were shorter for longer SOAs (Fig. 5B). (Nelson and Palmer obtained similar SOA effects.) RTs in the 0-ms SOA condition were longer than those in all other SOA conditions, $p$ s $<.001$, which did not differ significantly from each other, $p \mathrm{~s}>$.32. Region Type and SOA did not interact, $F(3,33)=$ $1.88, p=.151 .^{3}$

\section{Experiment $1 B$}

Accuracy Accuracy was low in Experiment 1B, when the target was exposed only for $80 \mathrm{~ms}$ and then masked. Accordingly, rather than analyzing response speed, we used

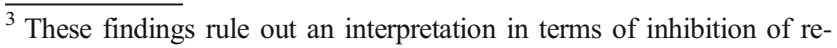
turn - that is, that attention was first drawn to the convex side, and then withdrawn, whereupon it was then harder to return to that side to attend to the red letter. Had this been the case, the advantage for targets on the concave side of the border would have been larger at longer SOAs.
}

$d^{\prime}$ to analyze participants' sensitivity to the differences between the two targets.

d' analysis We submitted the $d^{\prime}$ 'scores to an ANOVA with two within-subjects factors: Region Type (convex vs. concave) and $\operatorname{SOA}(0,150,250$, or $500 \mathrm{~ms})$. The main effect of Region Type was significant, $F(1,11)=7.81, p<.02$, but the $d^{\prime}$ effects were in the opposite direction from that reported by Nelson and Palmer (2007), replicating the pattern observed in Experiment 1A: Participants were more sensitive to targets shown on the concave (ground) than on the convex (figure) side $(d$ 's $=0.98$ and 0.80 , respectively; Fig. $6 \mathrm{~A})$. A main effect of SOA was also observed, SOA, $F(3,33)=36.90, p<.001$ : Participants discriminated targets more accurately at longer than at shorter SOAs (Fig. 6B). Pairwise comparisons indicated that sensitivity was lower in the 0 -ms SOA condition than in the other SOA conditions, $p \mathrm{~s}<.001$, and lower in the 150 $\mathrm{ms}$ condition than in the 250 -ms condition, $p<.04$. Sensitivity in the 250-ms and 500-ms SOA conditions did not differ, $p \mathrm{~s}>$ .60. Region Type and SOA again did not interact, $F(3,33)=$ $1.96, p=.138$.

\section{Discussion}

In Experiment 1, using eight-region displays, we found no evidence that attention is automatically allocated to convex figures or to the figural property of convexity. Instead, participants identified the target faster (Exp. 1A) and more accurately (Exp. 1B) in these eight-region displays when it was located on the concave region closest to fixation, rather than on the 


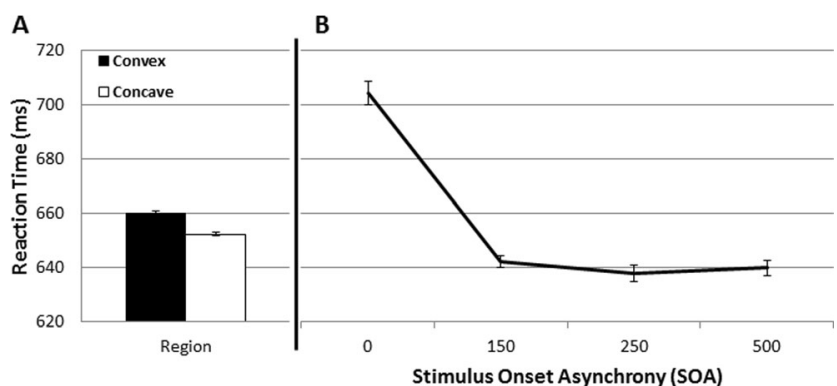

Fig. 5 Experiment 1A. (A) Mean reaction times (RTs) by Region Type. (B) Mean RTs by SOA

convex region closest to fixation. This pattern of results is the opposite of what one would expect if attention were automatically drawn to the convex figure closest to fixation. Because convex regions are highly likely to be perceived as figures in front of a surface interpolated across the concave regions in eight-region displays (Goldreich \& Peterson, 2012; Mojica \& Peterson, 2014), these results also indicate that results showing that attention is automatically drawn to near surfaces (e.g., Lester et al. 2009; West et al. 2013) do not generalize to figures perceived by virtue of the figural property of convexity. Yet the results of Experiment 1 are not anomalous, because the same pattern was observed when two different target exposure durations (unlimited in Exp. 1A; $80 \mathrm{~ms}$ with a mask in Exp. 1B) and two different response measures (RTs in Exp. 1A; $d^{\prime}$ in Exp. 1B) were employed.

One possible reason that we may have observed better accuracy and faster RTs for targets on concave rather than convex regions in Experiment 1 is that, because the borders between the convex and concave regions were perceived as the bounding contours of convex figures, they were perceived on the same depth plane as targets shown on the convex regions, but not as targets shown on the concave regions. Contours can mask nearby targets that appear to lie on the same depth plane: Lehmkuhle and Fox (1980) showed that depth separation reduces the masking effects of contours. The perceived depth separation between convex and concave regions may be enhanced in eight-region displays (cf. Goldreich \& Peterson, 2012) as compared to the two-region (bipartite) displays used by Nelson and Palmer (2007). As a consequence, targets on convex regions may have been masked more by the borders than were targets on the concave regions, leading to longer

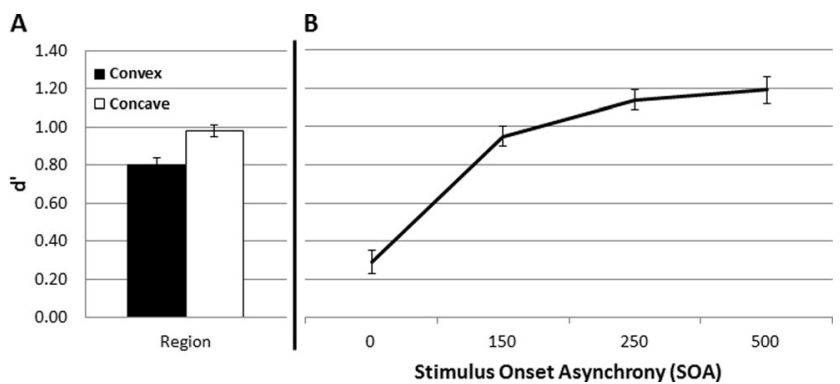

Fig. 6 Experiment 1B. (A) Mean $d^{\prime}$ by Region Type. (B) Mean $d^{\prime}$ by SOA
RTs and greater errors. Another possible explanation is that attention spread across the four convex figures in the eightregion displays, diluting any figural effect (cf. Roller, Mojica, Salvagio, \& Peterson, 2011).

Because the factors operating in eight-region displays may not be the same as those operating in two-region (bipartite) displays like those used by Nelson and Palmer (2007), in Experiment 2 we used bipartite displays like those in Fig. 7 to test whether attention can be automatically attracted to the figural property of convexity.

\section{Experiment 2}

The use of bipartite displays in Experiment 2 allowed us to test whether attention is automatically drawn to convex figures/the convex side of a border under conditions more similar to those Nelson and Palmer (2007) had employed to test familiarity. In addition, the use of bipartite displays with a central border that was convex on one side and concave on the other side allowed us to separately examine whether figures per se or properties that predict where figures are likely to lie in the visual field (figural properties) attract attention. This is because Peterson and Salvagio (2008) found that figures are perceived on the convex side of the central border in stimuli like these on only $57 \%-60 \%$ of trials; these percentages are statistically greater than chance, validating convexity as a figural property. ${ }^{4}$ Nevertheless, because the figure is perceived to be on the concave side of the central border on a sufficiently large percentage of trials ( $40 \%-43 \%)$, we can investigate whether discrimination RTs are faster for targets shown (a) on the side of the border that participants report perceiving as the figure (regardless of whether it is the convex or the concave side) or (b) on the side of the border where the figural property of convexity lies (regardless of whether or not participants perceive the figure on that side).

Accordingly, to allow this analysis, we asked the participants in Experiment 2 to make a second response after reporting the identity of the target: They reported whether the target had appeared on the side of the central border they perceived as the figure or on the side they perceived as the ground. If attention is drawn to figures, then participants' discrimination RTs should be faster for targets shown on the perceived figure rather than on the perceived ground, regardless of whether the perceived figure is convex or concave. Alternatively, if attention is drawn to the figural property of convexity, then participants' discrimination RTs should be

\footnotetext{
${ }^{4}$ This percentage is a pure index of the effect of convexity, unaided by another property that influences figure-ground perception in eight-region displays: The uniform fill in the concave regions in eight-region displays affords the interpretation that the concave regions are portions of a single surface that can be perceived as a ground to the convex figures (Goldreich \& Peterson, 2012; Mojica \& Peterson, 2014).
} 


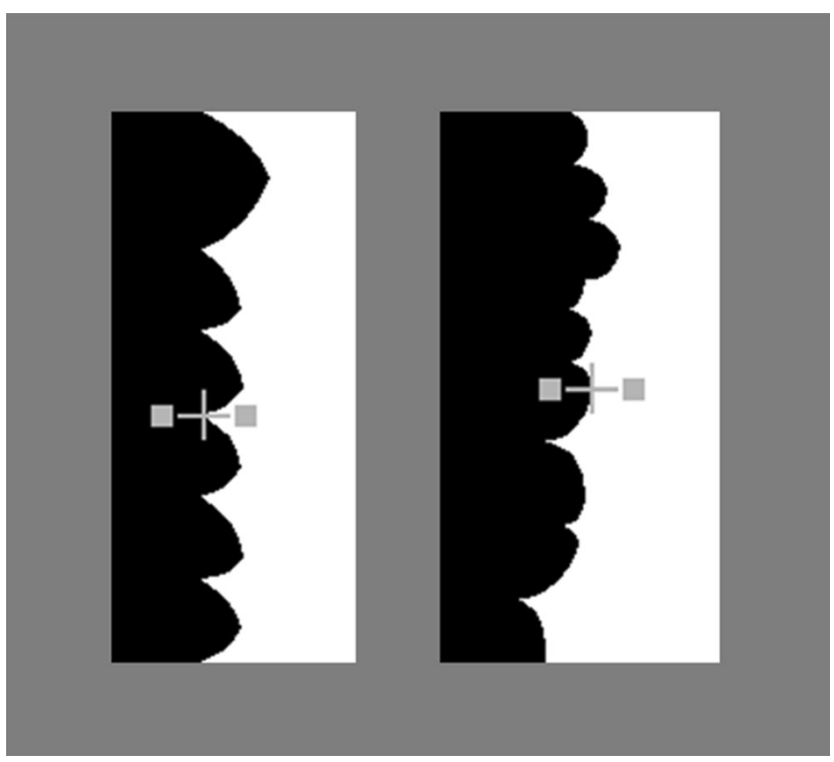

Fig. 7 Sample two-region displays, with a central border that is convex on one side (here, the left, black side) and concave on the other side (here, the right, white side). The display on the left shows targets aligned with a minimum of curvature defined from the convex side of the border, and the display on the right shows targets aligned with a maximum of curvature

faster for targets that appeared on the convex rather than on the concave side of the border, regardless of whether the participants perceived the convex side as the figure.

\section{Method}

\section{Participants}

A total of 32 (23 females, nine males) University of Arizona undergraduates participated in this experiment after giving informed consent; they took part to partially fulfill requirements for their introductory psychology class. All participants reported normal or corrected-to-normal visual acuity.

\section{Stimuli and apparatus}

The stimuli were bipartite displays divided into two equal-area regions by an articulated central border that sketched convex parts on one side and concave parts on the other side (Peterson \& Salvagio, 2008; Exp. 1). On the experimental trials, 64 unique displays were each shown once; on practice trials, another 32 unique displays were shown. Black/white fill and the left/right location of the convex side of the border were balanced across displays. ${ }^{5}$ All displays were $5.5^{\circ} \mathrm{H}$, and they averaged $3.0^{\circ} \mathrm{W}\left(\right.$ range $\left.=2.5^{\circ}-3.4^{\circ}\right)$. These displays were

\footnotetext{
${ }^{5}$ From here on, we refer to the convex or the concave side of the central border rather than to convex and concave regions because, in bipartite displays, only the central border is articulated.
}

centered on a medium gray backdrop (RGB 182, 182, 182), $17.7^{\circ} \mathrm{H} \times 22.8^{\circ} \mathrm{W}$.

A black fixation cross $\left(0.53^{\circ} \mathrm{H} \times 0.53^{\circ} \mathrm{W}\right.$; RGB, $\left.0,0,0\right)$ was presented before each trial. The location of this fixation cross was adjusted for each stimulus individually, such that it was aligned equally often with minima and maxima of curvature (defined from the convex side of the border) in the upcoming display. In this way, the central border locally enclosed targets on both the convex and the concave sides of the border equally often (see Fig. 7); hence, the border did not differentially mask targets on the convex versus the concave side. $^{6}$ The location of the fixation cross was shifted somewhat from trial to trial such that they were located where the central edge of the upcoming test display would appear. The targets were medium gray squares or circles (the squares were $0.24^{\circ}$ on a side, and the circles were $0.24^{\circ}$ in diameter; RGB 182 , $182,182)$. Targets appeared on the concave or the convex side of the central border, aligned with the horizontal arm of the fixation cross with their nearest edge one pixel away from where the horizontal arm of the fixation cross terminated. We chose this location because Nelson and Palmer (2007) had reported that attention effects were larger for targets located near the border than for those far from it.

A 21-in. Sony CRT monitor controlled by a personal computer was used to present the stimuli and record responses. Participants used a chinrest and viewed the monitor from a distance of $96 \mathrm{~cm}$. Responses were recorded via two custom button boxes, one with two horizontally arranged buttons and the other with two vertically arranged buttons. Participants used a foot pedal to initiate each trial and advance through the instructions. Visual stimulus presentation was controlled by DMDX (Forster \& Forster, 2003).

\section{Procedure}

Participants were instructed on the target discrimination task and the nature of figure-ground perception via computerdisplayed instructions. ${ }^{7}$ They were told that a black-andwhite display would appear briefly, and after a variable delay a brief alerting tone would be played, after which a target would appear on the left or the right side of the central border. They were instructed that their primary task was to identify the shape of the target as being either a circle or a square by using their dominant hand to press either the left or the right button on a custom-designed button box (assignment of the right/left buttons to the "circle"/"square" responses was balanced across participants). Participants were instructed that their secondary task was to indicate whether the target was

\footnotetext{
${ }^{6}$ Unmatched masking from the borders was not a factor in Experiment 1 because the targets were centered within the convex and concave regions. ${ }^{7}$ These instructions are available from the experimenters.
} 
positioned on the region they saw as the figure or on the region they saw as the background, by using their nondominant hand to press the top or the bottom button on a custom button box (the "figure" and "ground" button locations were also balanced across participants).

Each trial began with a central fixation cross. Participants were instructed to look at the fixation cross and to press the foot pedal when they were ready to begin the trial. The foot pedal press initiated the disappearance of the fixation cross and the appearance of a single bipartite display in the center of the screen; $60 \mathrm{~ms}$ later, a $1000-\mathrm{Hz}$ tone was played for $20 \mathrm{~ms}$. Immediately following the termination of the tone, a target shape appeared to the right or the left of the central border (thus, the test display-to-target SOA was $80 \mathrm{~ms}$ ). The two target shapes were equally likely to appear and appeared equally often on the left and right sides of the central border, which were equally often convex and concave. The target shape remained on the screen with the bipartite display for $100 \mathrm{~ms}$, and then both disappeared. The blank backdrop remained visible until participants made their discrimination response or until 3,000 ms had elapsed (a time-out was recorded in the latter case). Immediately after participants' target discrimination response, the prompt "figure or ground?" (or "ground or figure?") onset, centered on the screen, and remained visible for $500 \mathrm{~ms}$ (word order was balanced across participants). Participants' responses were recorded only if they occurred within $3,000 \mathrm{~ms}$ of the onset of the prompt.

Before the experimental trials, participants completed three sets of practice trials. An experimenter stayed in the room during the practice trials to answer questions. Participants first completed 16 practice trials for their primary task (target discrimination) alone. On these trials, they pressed a button on the button box positioned under their dominant hand to identify the target as a circle or a square. When they erred, feedback in the form of the word "WRONG" was displayed on the screen for $800 \mathrm{~ms}$. Next, they completed 16 practice trials for their secondary task alone. On these trials, they pressed a button on the button box under their nondominant hand to report whether the target shape had appeared on the side they perceived as being the figure or the ground; no feedback was given, since this was a subjective response. Different displays were used for practice on the primary and secondary tasks. Finally, participants completed 32 practice trials on which they made both primary- and secondary-task responses on each trial. The 32 stimuli used on the previous practice trials were intermixed here (although different target locations were used). After the practice trials, participants completed 64 experimental trials with unique displays. The participants viewed each bipartite display only once on the experimental trials, which they were left alone to complete.
Participants received feedback on their target discrimination performance on all trials. For correct trials, the word "correct" was displayed along with their RT; for incorrect trials, the word "wrong" was displayed for $800 \mathrm{~ms}$.

\section{Results}

We found no differences in $d^{\prime}$ for the targets shown on convex $\left(d^{\prime}=3.07\right)$ versus concave $\left(d^{\prime}=3.05\right)$ regions, $F(1,30)=$ 0.057, $p=.813$. In Experiment 2, using our preestablished criterion, we removed the data from one participant.

\section{Targets on the convex versus the concave side of the central border}

We first examined whether the figural property of convexity attracted attention by comparing the RTs for targets shown on the convex versus the concave side of the central border, regardless of whether participants reported perceiving the figure on the convex or the concave side. We found no differences: The mean RTs were 609.0 and $612.3 \mathrm{~ms}$ for targets shown on the convex versus the concave side of the central border, respectively. An ANOVA with one within-subjects factor (Side: convex vs. concave) did not show a main effect, $F(1,30)=$ $0.094, p=.761$. Thus, using bipartite displays in Experiment 2 , we found no evidence that attention is automatically drawn to the figural property of convexity.

\section{Targets on figures versus grounds}

For this analysis, we created a mean RT for all "figure" responses by averaging over trials on which participants reported that the target appeared "on the figure," regardless of whether these reports were made for targets shown on the convex or the concave side of the central border. We also created a mean RT for all "ground" responses by averaging over trials on which participants reported that the target appeared "on the ground," regardless of whether these reports were made for targets shown on the convex or the concave side of the central border. No differences were observed as a function of perceived figural status: The RTs for targets shown on figures versus grounds were 611.3 and $611.4 \mathrm{~ms}$, respectively, $F(1,30)=0.000, p=.993$. Thus, in Experiment 2, using bipartite displays as Nelson and Palmer (2007) had, we found no evidence that attention is automatically drawn to figures rather than to grounds when the figural property present was convexity.

The figure-versus-ground analysis was useful only to the extent that the figure-ground reports made after target identification responses were a reliable index of the perceived figure-ground assignments. To assess this reliability, we calculated the percentage of trials on which participants perceived 
the figure to be on the convex side of the central border in Experiment 2, and compared that to the percentage of trials on which Peterson and Salvagio's (2008) participants had perceived the figure on the convex side of the central border in the same displays when their only task was to report the perceived figure assignment. To calculate the percentage of trials on which the participants in Experiment 2 perceived the convex regions as figures, we added the "on figure" reports for targets shown on the convex side of the central border and the "on ground" reports for targets shown on the concave side of the border, ${ }^{8}$ and divided this sum by the total number of trials on which participants responded. The participants in Experiment 2 perceived the figure to be on the convex side of the central border on $55 \%(S E=0.02)$ of the trials, which was significantly greater than chance, $t(30)=2.76, p=.01$, and did not differ from the performance of Peterson and Salvagio's (2008) participants [who perceived the figure to be on the convex side of the central border of the same displays on $57 \%$ of trials, $F(1,49)=0.334, p=.566]$. We note that Peterson and Salvagio obtained similar results from participants who reported directly which side they perceived as figure or reported whether a probe appeared to lie "on" or "off" the region they perceived as figure, a task similar to the one that participants engaged in here. Accordingly, we conclude that the figure-ground reports in Experiment 2 were reliable indices of perception.

\section{Discussion}

In Experiment 2, like Nelson and Palmer (2007) we used bipartite displays; nevertheless, unlike Nelson and Palmer, we failed to find evidence that convex figures - or the figural property of convexity - attract attention. Does this mean that Nelson and Palmer's results are specific to familiar figures, or might a difference between our experiments and Nelson and Palmer's yet account for our different results? Three remaining differences were the sizes of the test displays, the numbers of target locations, and the temporal onset times. Nelson and Palmer used large displays $\left(>18^{\circ} \mathrm{high}^{9}\right)$ and intermixed at least four SOAs and 12 target locations in their experiments, whereas the Experiment 2 displays were smaller $\left(5.5^{\circ}\right.$ high), and the numbers of SOAs and of potential target locations were also smaller. If figures automatically attract attention, neither display size nor the numbers of target locations and onset times should matter, yet these factors may be responsible for the difference in results, because the participants in Nelson and Palmer's experiments may have been more

\footnotetext{
${ }^{8}$ We inferred that if the region on one side of the border was perceived as the ground (in this case, the concave region), the region on the other side of the border was perceived as the figure (in this case, the convex region).

${ }^{9}$ Nelson and Palmer (2007) did not report the size of their displays. However, the farthest targets were located $9^{\circ}$ above and below fixation, so we conclude that their displays were more than $18^{\circ}$ high.
}

uncertain of when and where the target would appear than were the participants in our experiments. Figural properties such as familiar configuration and convexity are priors regarding where objects lie in the visual field (e.g., Goldreich \& Peterson, 2012). It is well known that priors are given a larger weight in decision under conditions of uncertainty (e.g., Körding \& Wolpert, 2004). Hence, Nelson and Palmer may have observed faster and more accurate performance for targets shown on the familiar side of the border in their displays because figural properties were given higher weight in visual search under conditions of uncertainty. When observers have more information regarding the potential target locations, such information can be assigned greater weight than figural properties, and this may have occurred in Experiment 2, in which the targets appeared in one of two locations near the border at a single SOA.

The proposal that figural properties are priors that can guide search under conditions of uncertainty but that can be overcome by task-specific information is compatible with Shomstein's (2012; Drummond \& Shomstein, 2010; Shomstein \& Yantis, 2002, 2004) well-supported claim that the pattern of results initially taken to support automatic object-based attention occurs only under conditions of uncertainty regarding the target location. Rather than showing that figures are automatically attended, Nelson and Palmer's (2007) results may reflect the tacit reliance on priors such as the figural property of familiar configuration to guide search when there is uncertainty regarding where the targets will appear. On this alternative view, regularities in the environment can serve to prioritize search locations when there is no other information regarding the likely target locations, just as, ceteris paribus, locations within a cued object are prioritized in demonstrations of object-based attention. Others have shown that attention is guided by regularities in the environment (Jiang, Swallow, Rosenbaum, \& Herzig, 2013; Zhao, Al-Aidroos, \& Turk-Browne, 2013), although previous investigators have not explored the role of uncertainty.

\section{Experiment 3}

In Experiment 3, we investigated whether we could extend Nelson and Palmer's (2007) effects to convexity if we used large bipartite displays, 12 target locations, and four displayto-target SOAs. If we were to find that participants' target identification responses were faster for targets shown on the side of the border that participants perceived as the figure, rather than on the side they perceived as the ground, that would show that figures per se (rather than figural properties) are prioritized for search under conditions of uncertainty. On the other hand, if we were to find that participants' target identification responses were faster for targets shown on the convex rather than the concave side of the border, regardless 
of whether they perceived the convex side of the border as being the figure, that would show that the effects were due to the figural prior rather than to the perceived figure itself, and would be consistent with the proposal that locations in the visual field where figural properties are present are prioritized for search under conditions of uncertainty. Finally, a failure to replicate the effects found by Nelson and Palmer for either convex figures or the figural property of convexity in Experiment 3 would suggest an alternative interpretationthat Nelson and Palmer's results show that the specific property of familiarity attracts attention (cf. Christie \& Klein, 1995), rather than either figures or configural properties.

\section{Method}

\section{Participants}

A total of 45 (29 females, 16 males) undergraduate students from the University of Arizona participated in this study after giving informed consent; they took part in the experiment to partially fulfill the requirements for their introductory psychology class. All participants reported normal or correctedto-normal visual acuity. The data from 32 (22 females, ten males) participants were analyzed; the remaining 13 were eliminated because their mean score in at least one condition was more than two standard deviations from the condition mean. ${ }^{10}$

\section{Stimuli and apparatus}

The stimuli were bipartite black-and-white displays with an articulated central border with one convex and one concave side; the left/right location and the black/white fill of the convex side of the display were balanced, as in Experiment 2. A total of 208 unique bipartite displays were used in Experiment 3; 192 were viewed on the experimental trials, and 16 were viewed on the practice trials. The displays were all equal in height $\left(20^{\circ} \mathrm{H}\right)$ and varied in width, subtending a mean visual angle of $15.2^{\circ} \mathrm{W}$. The two-region displays were centered on a medium gray (RGB 127, 127, 127) backdrop $24.9^{\circ} \mathrm{H} \times 31.4^{\circ} \mathrm{W}$. Each experimental stimulus was presented twice, once with the target located on the convex and once with the target located on the concave side of the central border. A gray target letter (either an "x" or a "y," $0.2^{\circ} \mathrm{H} \times 0.2^{\circ} \mathrm{W}$; RGB 127, 127, 127; luminance $=5.8 \mathrm{ft}-\mathrm{L}$ ) was shown in one of 12 locations ( $\mathrm{six}$ on each side of

\footnotetext{
${ }^{10}$ This task was very difficult, due to the large size of the displays and the uncertainty regarding when and where the targets would appear. Also, the bipartite displays were black and white, and the target was medium gray. Nelson and Palmer's (2007) participants had lower error rates, perhaps because their medium gray targets appeared on red- and blue-colored regions (no information was given regarding their luminance), or perhaps because their displays included a black contour between the red and blue regions.
}

the central border, three above and three below fixation on each side), modeled as closely as possible on Nelson and Palmer's (2007) Experiment 3. The targets appeared at one of three distances from the central border $\left(0.6^{\circ}, 1.2^{\circ}\right.$, or $\left.2.4^{\circ}\right)$, above or below fixation on either the left or the right side of the display. These 12 locations lay along the circumference of a virtual circle with a $10^{\circ}$ diameter (as per Nelson and Palmer's, 2007, Exp. 3). Targets appeared at one of four SOAs after the onset of the display $(0,150,250$, or $500 \mathrm{~ms})$. The apparatus was identical to that used in Experiment 2, except that participants viewed the computer screen from a distance of $66 \mathrm{~cm}$.

\section{Design and procedure}

Each trial began with a central fixation cross $\left(0.78^{\circ} \mathrm{H} \times\right.$ $\left.0.78^{\circ} \mathrm{W}, \mathrm{RGB} 0,0,0\right)$, positioned at a location where the central edge of the upcoming bipartite display would appear (see Fig. 8). Participants were instructed to look at the fixation cross and to press the foot pedal when they were ready to begin the trial. The foot-pedal press initiated the appearance of a single bipartite display in the center of the screen. After a variable display-to-target SOA of $0,150,250$, or $500 \mathrm{~ms}$, a gray target letter appeared at one of the 12 target locations. The target letter was exposed for $80 \mathrm{~ms}$ and was followed by a blank gray screen that remained visible until response or for 3,000 $\mathrm{ms}$ (a time-out was recorded in the latter case). Participants identified the target letter as being either an " $\mathrm{x}$ " or a "y" by using their dominant hand to press the top or the bottom button on a custom button box (assignments of the buttons to " $x$ " and " $y$ " responses were balanced across participants). As in Experiment 2, after making their target identification response, participants reported whether the target had appeared on the region they saw as the figure or the ground.

The participants in Experiment 3 completed three sets of practice trials before the experimental trials (as had the participants in Exp. 2). There were 384 experimental trials.

\section{Results}

The discrimination sensitivities were equivalent for targets shown on the convex $\left(d^{\prime}=1.29\right)$ and the concave $\left(d^{\prime}=1.33\right)$ sides of the border, $F(1,31)=0.518, p=.48$.

\section{Reaction times}

We examined RTs as a function of both (a) whether the target appeared on the convex or the concave side of the border, regardless of whether it was perceived as figure or ground, and (b) whether the target appeared on the side of the central border that participants reported perceiving as figure, regardless of whether it was convex or concave. 


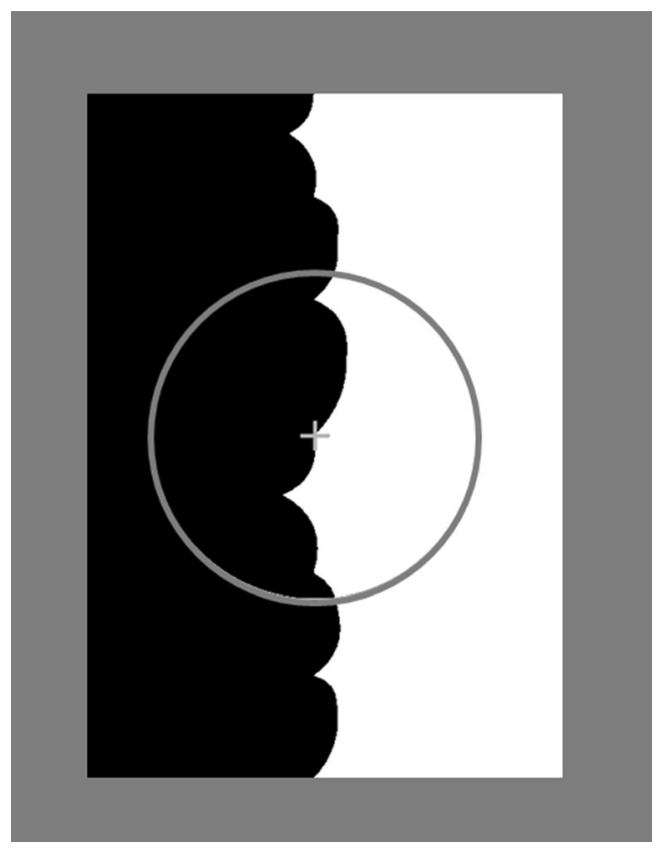

Fig. 8 Sample bipartite display used in Experiment 3. The 12 potential target locations appeared on the circumference of a circle, $10^{\circ}$ in diameter, shown in gray. The circle was used to choose target locations only; it was not present on experimental trials. The stimuli were $20^{\circ}$ high, with size and target location modeled closely on those of Nelson and Palmer (2007)

Role of the figural property of convexity An ANOVA was conducted on participants' RTs with three within-subjects factors: Side (convex or concave), Distance of the target from the central border $\left(0.6^{\circ}, 1.2^{\circ}\right.$, or $\left.2.4^{\circ}\right)$, and SOA $(0,150,250$, or $500 \mathrm{~ms})$. The results showed that the figural property played a role: Participants were faster to respond to targets shown on the convex $(631.8 \mathrm{~ms})$ than on the concave $(640.7 \mathrm{~ms})$ side of the border, as was revealed by a main effect of side, $F(1,31)=8.18$, $p<.01$; see Fig. 9A. Participants also responded faster to targets located at least $1.2^{\circ}$ from the border than to targets $0.6^{\circ}$ from the border $\left(0.6^{\circ}, 647.8 \mathrm{~ms} ; 1.2^{\circ}, 633.2 \mathrm{~ms} ; 2.4^{\circ}, 627.2 \mathrm{~ms}\right)$, as was shown by a main effect of distance, $F(2,62)=9.17, p<.001$. In addition, participants responded faster to targets that were shown at display-to-target SOAs greater than 0 (SOA $0 \mathrm{~ms}$, $689.0 \mathrm{~ms}$; SOA $150 \mathrm{~ms}, 609.4 \mathrm{~ms}$; SOA $250 \mathrm{~ms}, 614.3 \mathrm{~ms}$; SOA $500 \mathrm{~ms}, 632.3 \mathrm{~ms}$ ), as was shown by a main effect of SOA, $F(3,93)=65.74, p<.001$ (see Fig. 9C). There were no interactions among these factors, all $p \mathrm{~s}>.52 .{ }^{11}$

Role of the perceived figure As in Experiment 2, we calculated a mean RT for "perceived figures" by averaging over the "on figure" reports, regardless of whether those reports were made for targets shown on the convex or the concave side of the central border. We also created a mean RT for the perceived grounds by averaging over the "on ground" reports, regardless of whether these reports were made for targets

${ }^{11}$ The same patterns were evident in all participants.
A

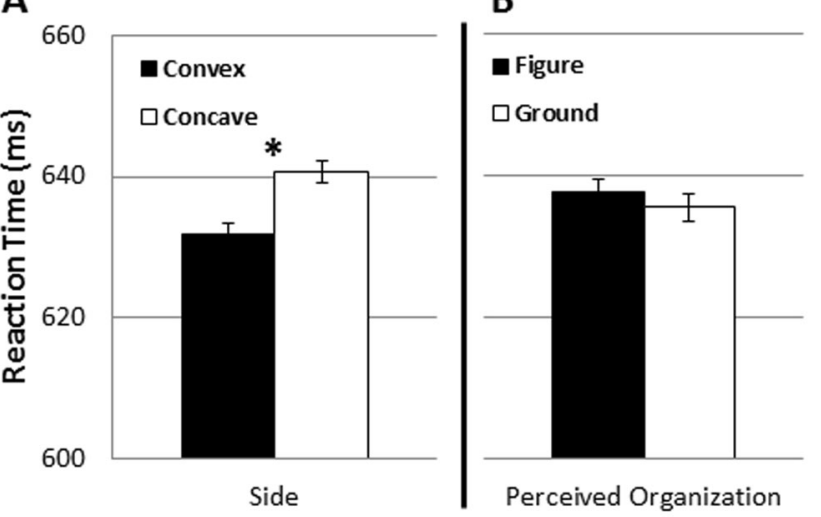

C

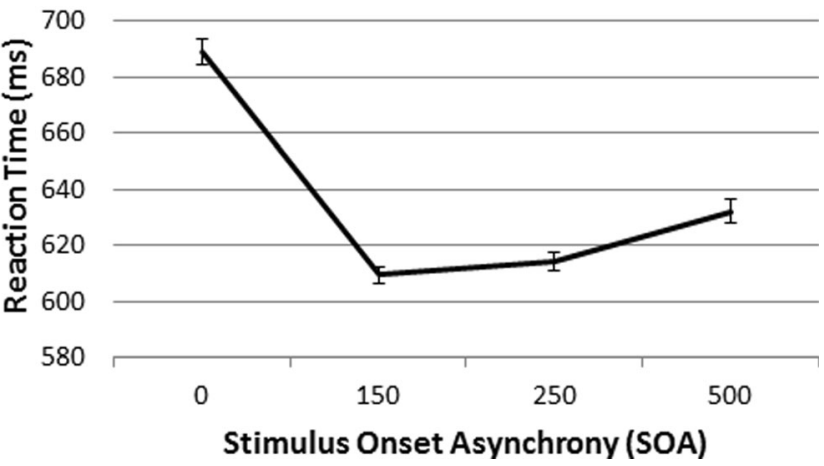

Fig. 9 Results of Experiment 3. (A) Reaction times (RTs) for targets on the convex versus the concave side of the border. (B) RTs by perceived figure-ground organization. (C) RTs by SOA

shown on the convex or the concave side of the central border. We did not find an attention advantage for targets shown on perceived figures rather than perceived grounds (figure, $637.6 \mathrm{~ms}$; ground, $635.5 \mathrm{~ms}), F(1,31)=0.319, p=.58$; see Fig. 9 B. ${ }^{12}$

\section{Figure-ground reports}

To determine whether participants' reports regarding which region they perceived as figure were affected by the fact that the figure-ground responses were their secondary task, we examined the percentage of trials on which they reported perceiving the figure on the convex side of the central border (calculated by adding the "on figure" reports for targets shown on the convex side of the central border and the "on ground" reports for targets shown on the concave side of the border, and dividing this sum by the total number of trials on which participants responded). Overall, convexity determined figure

\footnotetext{
$\overline{12}$ An analysis examining only trials on which participants perceived the figure on the convex side of the border and the ground on the concave side of the border also revealed no statistically significant differences in either RTs or $d^{\prime}(p \mathrm{~s}>.35)$. Note that this analysis was necessarily based on a smaller $N$ than the reported analyses, and hence is a less sensitive test.
} 
assignment on $59 \%$ of trials $(S E=0.03)$, which was significantly above chance, $t(31)=3.046, p=.006$. This percentage did not differ from the percentage reported by Peterson and Salvagio's (2008) participants (57 \%), for whom figureground reports were their primary task, $F(1,50)=0.204, p=$ .653. Therefore, we can be confident that participants' figureground reports in the present experiments were not affected by the fact that their primary task was target discrimination.

We note that target location did not affect the perceived figure reports: Participants' responses indicated that they perceived the figure on the convex side of the border on $60 \%$ of trials when the target appeared on that side ("on figure" responses), and on $57 \%$ of trials when the target appeared on the concave side of the central border ("on ground" responses), $F(1,31)=1.806, p=.189$; both of these responses indicate that convexity rather than target location determined figure assignment.

\section{Discussion}

In Experiment 3, we found that target identification responses were faster for targets shown on the convex rather than the concave side of the border. Like Nelson and Palmer (2007), we also found that participants responded faster to targets located farther from the border and at longer SOAs. Thus, Experiment 3 showed that Nelson and Palmer's results, obtained with the figural property of familiar configuration, generalize to another figural property - convexity. Taken together, however, the results of Experiments 1-3 show that, contrary to Nelson and Palmer's conclusion, the allocation of attention to the side of the border on which the figural property lies is not automatic; instead, such effects are observed only when the experimental conditions instantiate uncertainty regarding where and when the target will appear (e.g., very large displays, 12 potential targets locations, and four potential SOAs between the onset of the figure-ground display and the onset of the target, as in Exp. 3, but not smaller displays, with only two potential target locations close to fixation and a single SOA, as in Exp. 2).

Importantly, the results of Experiment 3 indicate that the figural property of convexity is what serves as the prior for visual search, not the perceived figure. Thus, we found support for the intriguing possibility raised by Nelson and Palmer (2007) that figural properties rather than perceived figures were responsible for the effects that they observed. We were able to test their hypothesis regarding figural properties because the figural property we used - convexity - determined where the figure was perceived in our bipartite displays on only $55 \%-59 \%$ of trials. Accordingly, we had enough trials on which the figure was perceived on the convex side of the central border to allow us to dissociate the effects of the figural property from the effects of the perceived figure. To do so, we asked participants to report whether the target had appeared on the side of the central border they saw as the figure or the side they saw as the ground. We showed that these figure-ground reports reliably indexed which region participants perceived as the figure, by showing that the percentage of trials on which the participants in Experiment 3 reported perceiving the figure on the convex side did not differ from that reported by Peterson and Salvagio's (2008) participants, whose only task was to report which region they perceived as figure. Moreover, Peterson and Salvagio had obtained the same results regardless of whether participants reported that they perceived a red probe as lying on the side they perceived as the figure or the ground, or whether participants directly reported that they perceived the black or the white side as the figure. Therefore, we consider the figure-ground reports made by the participants in Experiment 3 to be a valid assay of perceived figure-ground assignment.

How can different effects be obtained for figural properties and perceived figures? Perhaps in Experiment 3 search was initiated, while figure assignment was ongoing. According to the hypothesis that figural properties such as convexity are detected in a first pass of processing, but that figure assignment requires iterative cross-level interactions (see, e.g., Peterson \& Cacciamani, 2013), this claim is reasonable. According to this hypothesis, once convexity is detected, it can serve as the origin for search, even while figure assignment is being determined.

It is interesting to note that, although the participants in Experiment 3 discriminated targets shown on the convex side of the central border faster than targets shown on the concave side, they were no more likely to perceive the figure on the convex side when targets appeared there than when targets appeared on the concave side. Nor were they more likely to perceive the figure on the convex side of the central border in Experiment 3 than in other experiments in which search was not involved (cf. Peterson \& Salvagio, 2008). Thus, the form of attention used in the service of search in these experiments did not affect figure assignment. This is surprising in the context of other evidence showing that, ceteris paribus, attended regions are more likely to be perceived as figures than are unattended regions (Baylis \& Driver, 1995; Vecera, Flevaris, \& Filapek, 2004). Our finding suggests the interesting hypothesis that the attention used for prioritization differs from that allocated in experiments that have shown effects of attention on figure assignment. Investigating this hypothesis lies outside the scope of the present article, however.

\section{Experiment 4}

Taken together, Experiments 2 and 3 showed that Nelson and Palmer's (2007) results extend to convexity when the test displays are large and the target locations and timing are unpredictable, but not when displays are smaller and the target 
location and timing are more predictable. Our results are consistent with prioritization hypotheses proposed by others (e.g., Eckstein, 2011; Shomstein \& Yantis, 2002, 2004), in which different factors can serve as the basis for search prioritization. We showed that figural properties can serve as the starting point for search when target locations are uncertain (Exp. 3), but that the prioritization can be changed when target locations are more predictable (as in Exp.2). Nevertheless, it remained possible that the change from the small displays in Experiment 2 to the large displays in Experiment 3 alone accounted for the different results we obtained in those two experiments. Therefore, it was important to test whether the sizes of the displays per se, or rather uncertainty regarding spatiotemporal target appearance, underlay the effects we observed. We did so in Experiment 4 using bipartite displays like those used by Nelson and Palmer, in which the figural property of familiar configuration favored one side as figure. This allowed us to examine the role of uncertainty while holding size constant, and at the same time, to examine whether the uncertainty hypothesis applies to the figural property of familiar configuration as well as to the figural property of convexity. It is possible, for instance, that the property of familiar configuration automatically attracts attention, whereas convexity does not.

Accordingly, in Experiments 4A and 4B we used large $\left(\sim 20^{\circ}\right.$ high) bipartite displays in which a portion of a familiar configuration was sketched on one side of the central border. In Experiment 4A, the target could appear in any of 12 target locations (Fig. 10, left panel) at four stimulus-to-target SOAs; hence, spatiotemporal uncertainty regarding target appearance was high, as it was in Experiment 3 and in Nelson and Palmer's (2007) experiments. The experiment included a large number of trials, similar to Nelson and Palmer's experiment. In Experiment 4B, the target could appear in only four locations (Fig. 10, right panel), two on either side of the central border, and at only one SOA; hence, the spatiotemporal uncertainty regarding target appearance was low, as in Experiment 2. If uncertainty regarding when and/or where the target appears is critical for finding faster responses for targets on the side of the border where the figural cue lies, then we should replicate Nelson and Palmer's findings in Experiment 4A but not in Experiment 4B. On the other hand, if attention is automatically allocated to the side of the border where a figural cue is present when large displays are used, or if familiar configuration automatically attracts attention (even if convexity does not), then participants' discrimination responses should be faster for targets shown on the familiarconfiguration side of the central border in both Experiments $4 \mathrm{~A}$ and $4 \mathrm{~B}$.

Nelson and Palmer (2007) manipulated the orientation of their displays to test whether familiar configurations behaved differently when they were presented in an upright versus an inverted orientation; they did not always observe differences as a function of stimulus orientation. In Experiment 4, we also manipulated the orientation of the test displays, to make our test conditions as similar to theirs as possible.

\section{Method}

\section{Participants}

The participants were University of Arizona undergraduate students who took part in the experiment to partially fulfill requirements for their introductory psychology class; they gave informed consent before participating. All participants reported normal or corrected-to-normal visual acuity. A total of 43 (29 females, 14 males) participants took part in Experiment 4A; the data from 31 (21 females, ten males) participants were analyzed (the data from 11 of the remaining participants were removed because their RTs were more than two standard deviations longer than the mean in at least one condition; and the data from one participant were removed because she made so many errors that stable cell means could not be calculated). ${ }^{13}$ A total of 32 (20 females, 12 males) participants took part in Experiment 4B; the data from 30 (19 females, 11 males) participants were analyzed (the data from the remaining two participants were removed because their RTs were more than two standard deviations longer than the mean in at least one condition).

\section{Stimuli and apparatus}

In Experiment 4A, we attempted to replicate the experimental conditions employed by Nelson and Palmer (2007) as closely as possible. The stimuli were 16 bipartite figure-ground stimuli in which a central border dividing the displays into two equal-area regions sketched a portion of a familiar object on one side (see Fig. 10). The opposite (complementary) side of the border did not suggest anything familiar. Of these 16 stimuli, eight were viewed during experimental trials, and the remaining eight were viewed during practice trials. The central borders of the eight experimental-trial stimuli suggested portions of the following well-known objects on one side: bell, face, guitar, hydrant, lamp, pineapple, tree, and eagle. The central borders of the eight practice stimuli suggested portions of the following well-known objects on one side: apple, bulb, flower, house, milk can, pear, seahorse, and wrench. (In pretesting, pilot participants asked to name the objects suggested by these displays showed high between-subjects agreement on the identities of the well-known objects, and low

\footnotetext{
${ }^{13}$ Like Experiment 3, this experiment was difficult due to the large size of the displays, the spatiotemporal uncertainty regarding the targets, and potentially the low contrast of the medium gray target with the black/ white regions on which it appeared.
} 


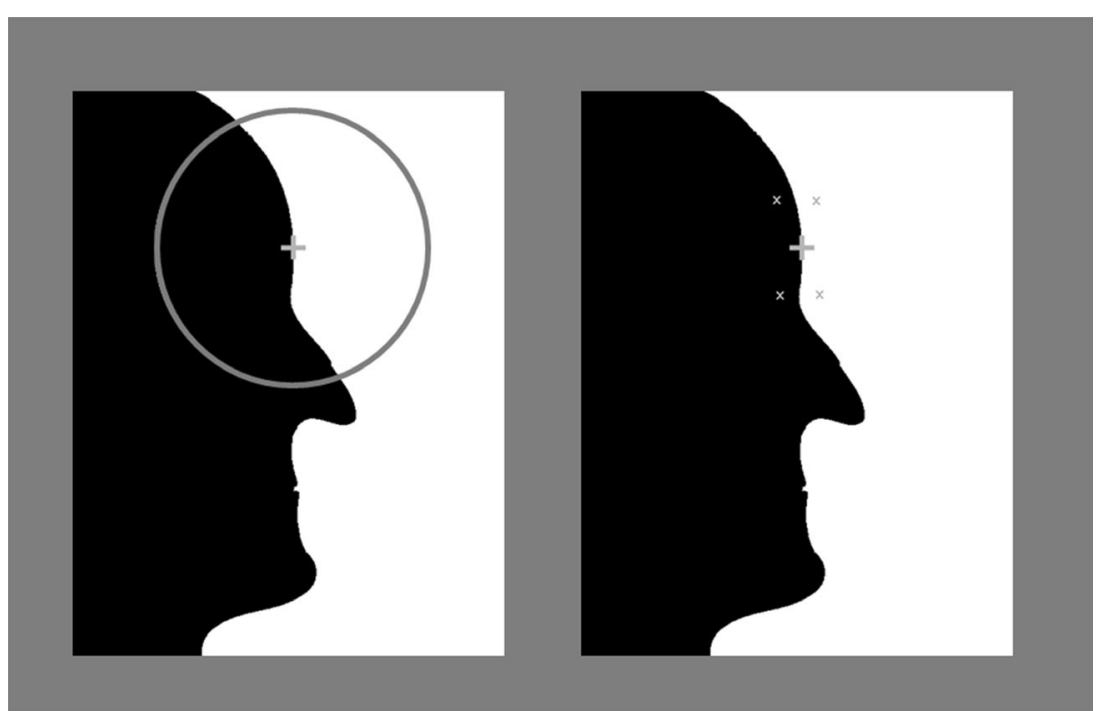

Fig. 10 (Left) Sample stimulus and a sample $10^{\circ}$-diameter circle on which the 12 target locations used in Experiment 4A were located. The location of the fixation cross varied somewhat for the various displays. It was always positioned at the location where the central edge of the upcoming bipartite display would appear, and such that six target locations appeared on each side of the central border. Hence, the locations of the targets with respect to the fixation cross were the same from one display to another, although the fixation cross was not always located at the center of the upcoming display. Here, the fixation cross is

between-subjects agreement regarding the complementary sides; see, e.g., Gibson \& Peterson, 1994; Peterson, Gerhardstein, Mennemeier, \& Rapcsak, 1998). The two sides in our stimuli were black and white, whereas in Nelson and Palmer's experiments theirs were blue and red, separated by a black central border. Eight variants of each stimulus were made, in which the side of the familiar configuration was swapped, the black/white fills on the familiar-configuration side of the border were swapped, and the familiar configuration was portrayed in its upright versus its inverted orientation. The displays were $20.1^{\circ} \mathrm{H}$ and varied in width, subtending a mean visual angle of $13.7^{\circ} \mathrm{W}$ (range $11.30^{\circ}-16.2^{\circ}$ ). The apparatus was identical to that used in Experiment 3.

The stimuli and apparatus used in Experiment 4B were the same as those in Experiment 4A, except that there were only four target locations: The target letters could appear either $1.7^{\circ}$ above or below fixation on either the familiar side or the complementary side of the central border $\left(0.6^{\circ}\right.$ from the border). For a given stimulus, the location of the fixation cross used in Experiment 4B was matched to that used in Experiment 4A. See Fig. 10, right panel. The display-to-target SOA was $80 \mathrm{~ms}$.

\section{Procedure}

The design and procedure used in Experiment 4 were similar to those used in Experiment 3, except that, on each trial, participants reported only whether the target letter was an " $x$ " or a "y." In Experiment 4A, the display-to-target SOA was 0, 150, shifted above the center of the display, but that was not always the case. (Right) Sample stimulus and four target locations used in Experiment 4B. The familiar configurations are in black on the left of the central border in these displays (the location and contrast polarity of the familiar configurations were balanced in the experiment). The familiar configuration is a face profile in both stimuli. The targets were medium gray; target size and the line thickness of both the fixation cross and the targets are increased here for visibility

250 , or $500 \mathrm{~ms}$. In Experiment 4B, the display-to-target SOA was always $80 \mathrm{~ms}$.

In both Experiments 4A and 4B, participants completed 32 practice trials before the experimental trials. No feedback was given during practice or the experimental trials. None of the figure-ground displays presented on practice trials was presented on the experimental trials. At the conclusion of the practice trials in Experiment 4A, participants completed 1,536 experimental trials ( 24 trials with each of the eight variants of each of the eight stimuli). In Experiment 4B, participants completed 512 experimental trials (eight trials with each of the eight variants of each of the eight stimuli). In both experiments, figure-ground perception was assessed after the completion of all experimental trials by presenting each stimulus singly on the screen and asking participants to record whether they perceived the figure on the left or the right side of the central border. The purpose was to determine whether participants had perceived the familiar shape as figure during the experiment. Nelson and Palmer (2007) had used postexperiment figure-ground ratings in four of their five experiments.

\section{Results}

\section{Experiment $4 A$}

Participants' sensitivity indices ( $d$ 's) did not differ for targets shown on the familiar-configuration side of the central border 
$\left(d^{\prime}=1.28\right)$ or the complementary side $\left(d^{\prime}=1.23\right), F(1,30)=$ $3.31, p=.08$.

Reaction times The RTs were subjected to an ANOVA with four within-subjects factors: Side (familiar configuration or complement), Orientation of the familiar configuration (upright or inverted), display-to-target $\operatorname{SOA}(0,150,250$, or $500 \mathrm{~ms})$, and Distance of target from central border $\left(0.6^{\circ}\right.$, $1.2^{\circ}$, or $\left.2.4^{\circ}\right)$. Participants' discrimination responses were faster for targets shown on the familiar-configuration side of the central border $(592.8 \mathrm{~ms})$ than on the complementary side $(600.8 \mathrm{~ms})$, as revealed by a main effect of Side, $F(1,30)=$ $15.02, p=.001$ (see Fig. 11A). Discrimination responses were faster for targets shown on upright $(594.8 \mathrm{~ms})$ rather than inverted ( $598.8 \mathrm{~ms}$ ) displays, as evidenced by a main effect of Orientation, $F(1,30)=6.64, p<.02$, as is shown in Fig. 11B. A main effect of Distance, $F(1.59,47.65)=29.90$, $p<.0001$, showed that RTs decreased significantly as the distance between the target and the border increased (see Fig. 11C). The ANOVA also showed a main effect of SOA, $F(2.07,62.01)=161.74, p<.0001$ : RTs were shorter when the display-to-target SOA was $150 \mathrm{~ms}$ or longer than when the target and display onsetted together (see Fig. 11D). Orientation and Side did not interact, $p>.66$ : The orientation effect was the same for both the side of the central border where the familiar configuration lay and the complementary side, replicating the results reported by Nelson and Palmer (2007). Neither Distance and Side nor SOA and Distance interacted, $p \mathrm{~s}>.06$. There was an interaction between side and SOA, $F(3,90)=4.69, p<.005$, indicating that the advantage for targets on the familiar-configuration side of the border relative to the complementary side was larger in the 0 -ms and 250-ms SOA conditions (16.6 and $11.8 \mathrm{~ms}$, respectively) than in the $150-\mathrm{ms}$ and $500-\mathrm{ms}$ SOA conditions $(2.7 \mathrm{~ms}$ and $0.9 \mathrm{~ms}$, respectively).

\section{Experiment 4B}

The results of Experiment 4B, in which there was little spatiotemporal uncertainty regarding target appearance, are very different from those of Experiment 4A. An ANOVA was conducted with two within-subjects factors: Side and Orientation. (Neither SOA nor distance from the border was a factor in Exp. 4B, because all targets were equidistant from the central border and appeared $80 \mathrm{~ms}$ after the display onset.) Neither main effect was statistically significant [side, $F(1,29)=0.10$, $p>.75$ (mean RT for targets on the familiar-configuration side $=517.6 \mathrm{~ms}$, mean RT on complementary side $=518.9 \mathrm{~ms}$ ); orientation, $F(1,29)=1.20, p>.27$ (mean RT for targets on upright displays $=517.8 \mathrm{~ms}$, mean RT on inverted displays $=$ $514.8 \mathrm{~ms})$ ], nor was the interaction between orientation and side, $F(1,29)=1.06, p>.30$.
Figure-ground reports When questioned after the experimental trials, the participants in Experiment $4 \mathrm{~A}$ reported that they had perceived the figure on the familiar-configuration side of the central border on an average of $86 \%$ of the trials $(S E=0.03)$, regardless of orientation (upright $=90 \%$, inverted $=82 \%$ ), which was significantly above chance, $t(31)=34.41$, $p=.001$. The participants in Experiment $4 \mathrm{~B}$ reported that they had perceived the familiar-configuration side as figure on an average of $85 \%$ of the trials $(S E=0.03)$, regardless of orientation (upright $=91 \%$, inverted $=82 \%$ ), which was again significantly above chance, $t(31)=33.68, p=.0001$.

\section{Discussion}

In Experiment 4A, we replicated Nelson and Palmer's (2007) results using conditions that closely mimicked theirs: very large $\left(\sim 20^{\circ}\right.$ high $)$ displays, 12 target locations, and four display-to-target SOAs - conditions that we argue induced uncertainty regarding when and where the target would appear. Under these conditions, participants' discrimination RTs were faster for targets shown on the side of the border where the familiar configuration lay than for targets shown on the opposite side.

In Experiment 4B, in which the displays were as large as in Experiment 4A, but the target location and onset time were predictable, participants were no faster to detect targets that appeared on the familiar-configuration side of the border than to detect those on the opposite side. Thus, the results of Experiment 4 show that uncertainty regarding when and where the target will appear is necessary to obtain the RT advantage that Nelson and Palmer (2007) reported for familiar configurations; the mere presence of a familiar configuration on one side of the border is insufficient, as is the mere use of large displays. Thus, together with the results of the previous experiments, Experiment 4 showed that figural cues do not automatically attract attention. Uncertainty regarding target location is necessary to observe effects of figural properties.

In Experiment 4, we did not attempt to separately assess whether the effects were due to the prioritization of the perceived figure or the figural property. This was because familiar configurations are perceived as figure on a larger proportion of trials than are convex regions ( $\sim 5 \%$ vs. $57 \%$ ), and we would not have had much sensitivity to separately assess the role of the figural property. Moreover, Experiment 4A was very long (participants performed 1,536 experimental trials), because we were replicating Nelson and Palmer's (2007) experiment as closely as possible; adding a figure-ground report after each trial would have made the experiment prohibitively long.

We note that under conditions of uncertainty in Experiment 4A, although participants did detect targets faster when the targets appeared on upright rather than inverted displays, the orientation effect did not vary with whether the target was on the familiar-configuration or the complementary 
A

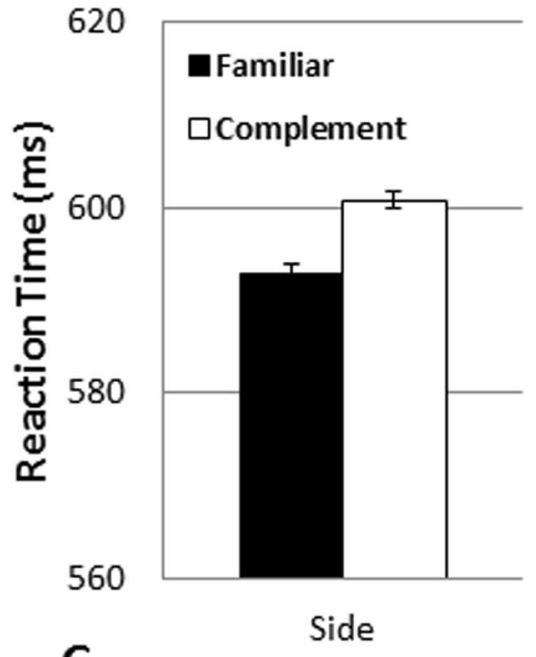

B

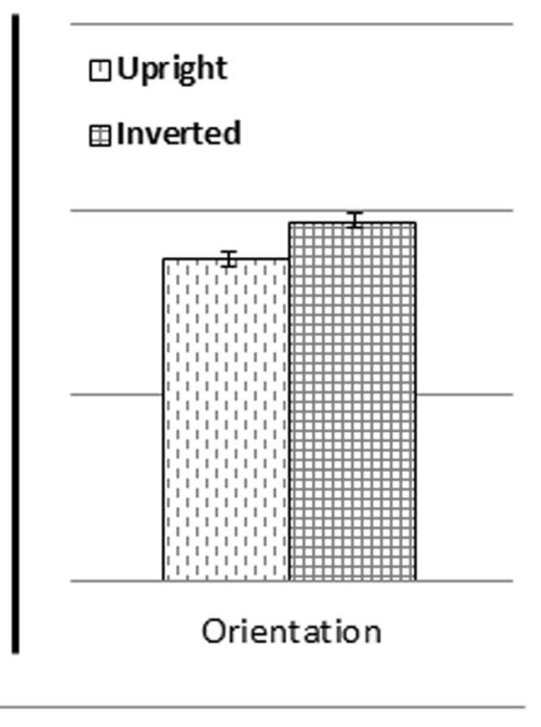

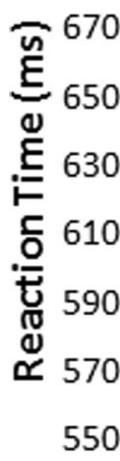

C 690

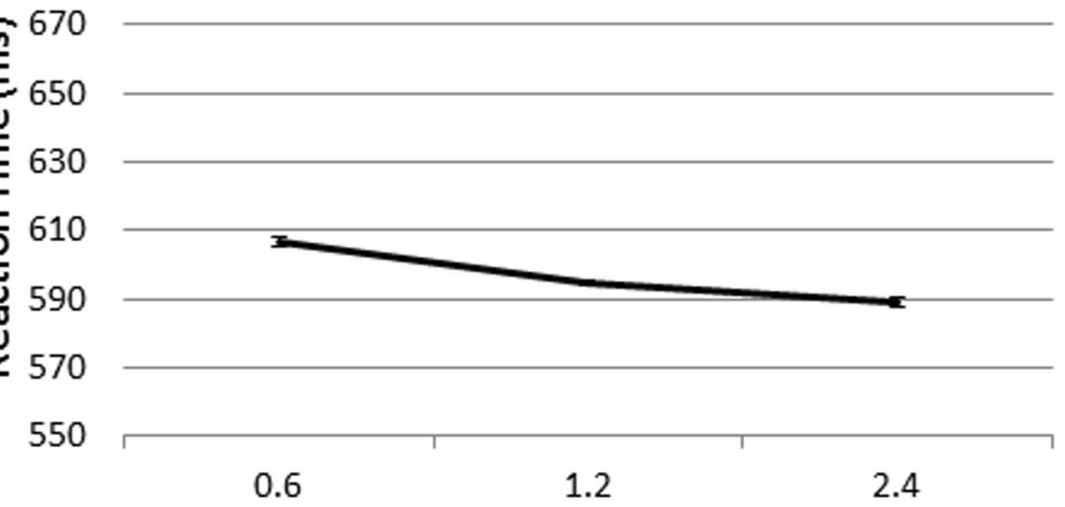

Target Distance from Border (degrees)

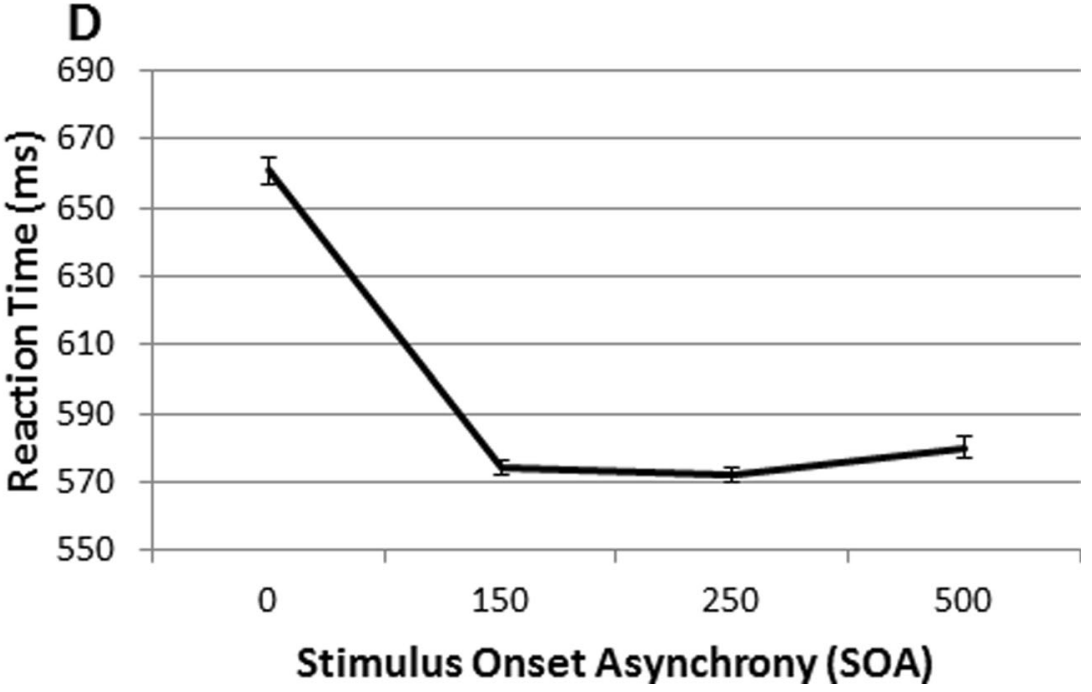

Fig. 11 Results of Experiment 4A. (A) Reaction times (RTs) for targets on the familiar versus the complementary side of the border. (B) RTs by orientation. (C) RTs by distance from border. (D) RTs by SOA

side of the central border. This finding replicates Nelson and Palmer's (2007) findings and stands in contrast to many demonstrations that familiar configuration exerts a larger influence on figure assignment when familiar objects are 
suggested in their upright rather than in an inverted orientation (e.g., Gibson \& Peterson, 1994; Peterson \& Gibson, 1994a, 1994b; Peterson et al. 1991). The absence of an orientationdependent effect of the familiar configurations in Experiment 4 and in some of Nelson and Palmer's experiments suggested to us the intriguing possibility that the initial access to memories of familiar objects by inverted familiar configurations in the first pass of processing may be sufficient to stimulate use of the figural property to prioritize search under conditions of uncertainty, even though it is not sufficient to exert as large an influence on figure assignment as is observed for upright displays (see also Cacciamani, Ayars, \& Peterson, 2014). This possibility will be interesting to explore in future experiments, although it is beyond the scope of the present article. Alternatively, the many repetitions of the stimuli in upright and inverted orientations may have underlain the absence of an orientation-dependent effect of familiar configurations. In the experiments demonstrating that familiar configurations exerted a larger influence on figure assignment in their upright than in their inverted orientations, the stimuli were shown only once upright and once inverted, or were shown in one orientation only.

\section{General discussion}

We examined whether attention is automatically allocated to figures or to figural properties, as Nelson and Palmer (2007) had claimed, on the basis of experiments in which they found that participants were faster and more accurate to detect and discriminate targets shown on the side of the central border of a bipartite display that portrayed a familiar configuration. Familiar configuration is a figural property in that, ceteris paribus, figures are more likely to be perceived on the side of a border that portrays a familiar object than on the complementary, unfamiliar, side (for reviews, see Peterson, 1994; Peterson \& Skow-Grant, 2003). Our approach was to test whether we would obtain the same effects with the figural property of convexity. In Experiment 1, we used eight-region displays comprising alternating convex and concave regions because convex regions are substantially more likely to be perceived as figures in such displays than in bipartite displays like those Nelson and Palmer had used. Targets appeared on either the central convex region or the central concave region of these displays. We found no evidence that attention was automatically allocated to the convex regions. Instead, participants detected targets faster and more accurately when the targets appeared on the central concave region rather than the central convex region. We hypothesized that in eight-region displays, in which depth perception is likely to be greater than in two-region displays, the border between the convex and concave regions was more likely to be perceived as the bounding contour for convex figures/objects. Previous research had revealed that contours mask targets perceived to be on the same depth plane (e.g., Lehmkuhle \& Fox, 1980); therefore, the targets on convex regions may have been masked by the contours. Alternatively, attention may have been spread across four convex figures/objects (cf. Roller et al. 2011), thereby diluting any effects of attention on targets appearing on the convex region closest to fixation. Accordingly, for the remaining experiments, we used bipartite displays.

In Experiments 2 and 3, we used bipartite displays in which a central border suggested a portion of an object with convex parts on one side and a portion of an object with concave parts on the other side. In Experiment 2, the displays were small $\left(5.5^{\circ}\right.$ high $)$ and there were only two possible target locations. After participants had made their target identification response, they reported whether the target had appeared on the side of the central border they had perceived as the figure or on the side they had perceived as the ground. To determine whether any differences that emerged should be attributed to the figural property of convexity (regardless of whether the figure was perceived on the convex side of the border) or to the perceived figure (regardless of whether it was convex or concave), we analyzed RTs as a function of both whether the target had appeared on the convex or the concave side of the central border and whether the target had appeared on the side participants had reported perceiving as the figure versus the ground. In Experiment 2, we found no evidence of faster discrimination RTs for targets shown on the convex rather than the concave side of the border or on the perceived figure versus the ground.

In Experiment 3, we used the same presentation conditions tested by Nelson and Palmer (2007): Our displays were large $\left(20^{\circ}\right.$ high $)$, and there were 12 possible target locations and four display-to-target SOAs. Here, we observed faster discrimination RTs for targets shown on the convex side rather than the concave side of the border (regardless of whether the figure was perceived on that side). No RT differences emerged as a function of which side participants perceived as the figure. Thus, Experiment 3 showed that the use of large displays and spatiotemporal uncertainty regarding target location are necessary for observing results for convexity like those Nelson and Palmer observed for familiarity. Furthermore, Experiment 3 revealed that enhanced discrimination occurs for targets shown on the side of the border where the figural property of convexity lies, not on the side of the border where the perceived figure lies.

In Experiment 4, we separately investigated the role of the large display size and that of spatiotemporal uncertainty by using displays in which the figural property was familiar configuration - the property tested by Nelson and Palmer (2007). We replicated their effects under conditions of spatiotemporal uncertainty (12 potential target locations, four SOAs; Exp. 4A), but not under conditions in which the target could appear in one of only four locations (Exp. 4B). 
Taken together, the experiments presented here demonstrate that, contrary to Nelson and Palmer's (2007) claim, attention is not automatically allocated to either figures or figural properties. According to the automatic view, one would expect to see enhanced discrimination of targets on figures (or on the side of a border where figural properties lie), even when there are only a few potential target locations. Instead, under these conditions, when bipartite stimuli were tested, the discrimination speed was approximately the same for targets, regardless of whether they appeared on the convex or the concave side of the border, on the perceived figure or the perceived ground. (Also recall that, in Exp. 1 in which eightregion displays were used, discrimination speed and accuracy were better for targets shown on concave rather than convex regions.)

The present experiments also show that under conditions of uncertainty, figural properties rather than perceived figures are prioritized for search. Nelson and Palmer (2007) introduced the intriguing question of whether figural properties per se draw attention, questioning whether "the same cues that cause one side of a contour to be perceived as figure (meaningfulness, surroundedness, symmetry, etc.) also draw attention to that side" (p. 383). They did not try to separate figural properties from perceived figures, however. Indeed, they used the term "figure" to refer to both, stating, "in the interest of brevity, we will refer to one side as figure rather than 'the region influenced by shape cues to be most often perceived as figure." (p. 383). The use of the term "figure" may lead readers astray. Moreover, in both their Abstract and their General Discussion Nelson and Palmer liken their effects to demonstrations that attention is automatically drawn to the onset of a new object (Yantis \& Hillstrom, 1994; Yantis \& Jonides, 1984) stating, "Thus, when a bipartite figure-ground display, such as those used in the present experiments, suddenly appears, only one new object actually appears - namely, the figure" (Nelson \& Palmer, 2007, p. 391). But in this claim the distinction between figural properties and figures is lost because a new object is present only after figure assignment has occurred. In the present experiments, we were able to separate figural properties from perceived figural status by using bipartite displays with one convex and one concave region in Experiments 2 and 3. Based on previous research, we expected that the figure would be perceived on the convex side of the border on approximately $60 \%$ of trials, leaving a large enough percentage of trials on which the convex side was perceived as the ground to allow us to separately assess effects of figures versus figural properties. Our results demonstrate that figural properties rather than perceived figures are prioritized in the service of search under conditions of uncertainty. Given that figural priors are environmental regularities these results are consistent with the hypothesis that, under conditions of uncertainty, priors that represent environmental regularities can inform behavior in general (Körding \&
Wolpert, 2004) and search in particular (Eckstein, 2011). Bisley and Goldberg (2010) propose that a priority map is represented in the lateral intraparietal region (LIP) and that at any given moment, attention is allocated on the basis of the activity in this priority map. Our results are the first to show that figural properties are represented in the priority map and can serve as the basis of search.

How important is it to distinguish whether figural properties or figures per se serve as the basis for search under conditions of uncertainty and whether these effects are automatic? We argue that a proper understanding of attention requires answers to these questions. In recent theory, figural properties are considered object priors. The present study extends the function of these priors beyond figure assignment into search. Moreover, figure assignment is a time-consuming process (e.g., Peterson \& Enns, 2005; Peterson \& Lampignano, 2003; Roelfsema, Lamme, Spekreijse, \& Bosch, 2000). The effects observed in the present article seem to take place before figure assignment is determined and are independent of the outcome - at least in bipartite displays in which the role of the figural property and the perceived figure can be separated as in Experiment 3. Therefore our results suggest that prioritization can be determined earlier in processing than would be required if the perceived figure per se biased search. As Shomstein (2012) has argued, when uncertainty is high, the brain utilizes all of the information in the environment to guide attention. Our research shows that it uses figural priors that are available early in processing over figures that are determined later in time. We hypothesize that prioritization can occur after an initial fast pass of processing identifies figural properties and their locations but before perceptual organization processes have determined which region is the figure.

Moreover, the finding that these effects are not automatic speaks to the flexibility of the prioritization system. As others have argued (Eckstein, 2011; Shomstein, 2012), when there is certainty regarding the locations of targets, attention can be restricted to important locations without interference from factors that putatively control attention automatically. In contrast, when certainty is reduced or absent, priors can guide attention.

The results of the experiments reported here go beyond evidence that regularities established during the course of an experiment attract attention (e.g., Jiang et al. 2013; Zhao et al. 2013). Here we showed that these effects extend to figural properties-environmental regularities that have been established during the lifetime of the participant. Moreover, we showed that effects of figural properties on target discrimination performance can be observed, but only under conditions of uncertainty. We note that previous experiments showing that environmental regularities attract attention had used situations in which the target location was uncertain. Similarly, the Gestalt factors of closure and good continuation were found to suffice for an object to capture attention 
(Kimchi et al. 2016), but these effects were again obtained when the target location was uncertain; it will be important to test whether those effects would also be obtained under conditions in which the target location was more certain. On the basis of our results and of Bayesian observer models, which assign greater weight to priors under condition of uncertainty, we predict that those results will also require conditions of uncertainty. In the future, it would also be interesting to separate the relative contributions of spatial and temporal uncertainty.

Finally, future research must solve the apparent inconsistency between evidence that both endogenously and exogenously oriented attention affect figure assignment (Baylis \& Driver, 1995; Vecera et al. 2004) and our results that have shown that attention allocated to figural properties in the service of target search does not affect figure assignment (recall that although participants were faster to detect targets shown on the convex rather than the concave ide of the border, they were not more likely to perceive the figure on the convex side of the border when targets appeared there rather than on the concave side). This finding suggests the interesting hypothesis that the form of attention deployed in the present experiments - what we and others have termed "prioritization"may be different from the forms of attention deployed in the previous work on figure-ground assignment.

Acknowledgments MAP acknowledges the support of NSF BCS 0960529 and ONR N00014-14-1-067.

\section{References}

Baylis, G. C., \& Driver, J. (1995). One-sided edge assignment in vision: 1. Figure-ground segmentation and attention to objects. Current Directions in Psychological Science, 4, 140-146.

Bisley, J. W., \& Goldberg, M. E. (2010). Attention, intention, and priority in the parietal lobe. Annual Review of Neuroscience, 33, 1-21. doi:10.1146/annurev-neuro-060909-152823

Cacciamani, L., Ayars, A. A., \& Peterson, M. A. (2014). Spatially rearranged object parts can facilitate perception of intact whole objects. Frontiers in Psychology, 5, 482. doi:10.3389/ fpsyg.2014.00482

Christie, J., \& Klein, R. (1995). Familiarity and attention: Does what we know affect what we notice? Memory \& Cognition, 23, 547-550. doi:10.3758/BF03197256

Drummond, L., \& Shomstein, S. (2010). Object-based attention: Shifting or uncertainty? Attention, Perception \& Psychophysics, 72, 17431755. doi:10.3758/APP.72.7.1743

Eckstein, M. P. (2011). Visual search: A retrospective. Journal of Vision, 11(5), 14. doi:10.1167/11.5.14

Egly, R., Driver, J., \& Rafal, R. D. (1994). Shifting visual attention between objects and locations: Evidence from normal and parietal lesion subjects. Journal of Experimental Psychology: General, 123, 161-177. doi:10.1037/0096-3445.123.2.161

Forster, K. I., \& Forster, J. C. (2003). DMDX: A Windows display program with millisecond accuracy. Behavior Research Methods, Instruments, \& Computers, 35, 116-124. doi:10.3758/BF03195503
Gibson, B. S., \& Peterson, M. A. (1994). Does orientation-independent object recognition precede orientation-dependent recognition? Evidence from a cuing paradigm. Journal of Experimental Psychology: Human Perception and Performance, 20, 299-316. doi:10.1037/0096-1523.20.2.299

Goldreich, D., \& Peterson, M. A. (2012). A Bayesian observer replicates convexity context effects. Seeing and Perceiving, 25, 365-395.

Hochberg, J. (1971). Perception I: Color and shape. In J. W. Kling \& L. A. Riggs (Eds.), Woodworth and Schlossberg's experimental psychology (3rd ed., pp. 395-474). New York: Holt, Rinehart \& Winston.

Jiang, Y. V., Swallow, K. M., Rosenbaum, G. M., \& Herzig, C. (2013). Rapid acquisition but slow extinction of an attentional bias in space. Journal of Experimental Psychology: Human Perception and Performance, 39, 87-99. doi:10.1037/a0027611

Jonides, J. (1981). Voluntary versus automatic control over the mind's eye's movement. In J. Long \& A. Baddeley (Eds.), Attention and performance $I X$ (pp. 187-203). Hillsdale: Erlbaum.

Kanizsa, G., \& Gerbino, W. (1976). Convexity and symmetry in figureground organization. In M. Henle (Ed.), Vision and artifact (pp. 25 32). New York: Springer.

Kimchi, R., Yeshurun, Y., Spehar, B., \& Pirkner, Y. (2016). Perceptual organization, visual attention, and objecthood. Vision Research, 126, 34-51. doi:10.1016/j.visres.2015.07.008

Kimchi, R., Yeshurun, Y., \& Cohen-Savransky, A. (2007). Automatic, stimulus-driven attentional capture by objecthood. Psychonomic Bulletin \& Review, 14, 166-172. doi:10.3758/BF03194045

Körding, K. P., \& Wolpert, D. M. (2004). Bayesian integration in sensorimotor learning. Nature, 427, 244-247.

Lehmkuhle, S., \& Fox, R. (1980). Effect of depth separation on metacontrast masking. Journal of Experimental Psychology: Human Perception and Performance, 6, 605-621. doi:10.1037/ 0096-1523.6.4.605

Lester, B. D., Hecht, L. N., \& Vecera, S. P. (2009). Visual prior entry for foreground figures. Psychonomic Bulletin \& Review, 16, 654-659. doi:10.3758/PBR.16.4.654

Mojica, A. J., \& Peterson, M. A. (2014). Display-wide influences on figure-ground perception: The case of symmetry. Attention, Perception \& Psychophysics, 76, 1069-1084. doi:10.3758/s13414014-0646-y

Nelson, R. A., \& Palmer, S. E. (2007). Familiar shapes attract attention in figure-ground displays. Perception \& Psychophysics, 69, 382-392. doi:10.3758/BF03193759

Peterson, M. A. (1994). Object recognition processes can and do operate before figure-ground organization. Current Directions in Psychological Science, 3, 105-111.

Peterson, M. A., \& Cacciamani, L. (2013). Toward a dynamical view of object perception. In S. J. Dickinson \& Z. Pizlo (Eds.), Shape perception in human and computer vision: An interdisciplinary perspective (pp. 445-457). London: Springer.

Peterson, M. A., \& Enns, J. T. (2005). The edge complex: Implicit memory for figure assignment in shape perception. Perception \& Psychophysics, 67, 727-740. doi:10.3758/BF03193528

Peterson, M. A., Gerhardstein, P. C., Mennemeier, M., \& Rapcsak, S. Z. (1998). Object-centered attentional biases and object recognition contributions to scene segmentation in left- and right-hemispheredamaged patients. Psychobiology, 26, 557-570.

Peterson, M. A., \& Gibson, B. S. (1993). Shape recognition contributions to figure-ground organization in three-dimensional displays. Cognitive Psychology, 25, 383-429.

Peterson, M. A., \& Gibson, B. S. (1994a). Must figure-ground organization precede object recognition? An assumption in peril. Psychological Science, 5, 253-259.

Peterson, M. A., \& Gibson, B. S. (1994b). Object recognition contributions to figure-ground organization: Operations on outlines and 
subjective contours. Perception \& Psychophysics, 56, 551-564. doi:10.3758/BF03206951

Peterson, M. A., Harvey, E. H., \& Weidenbacher, H. L. (1991). Shape recognition inputs to figure-ground organization: Which route counts? Journal of Experimental Psychology: Human Perception and Performance, 17, 1075-1089.

Peterson, M. A., \& Lampignano, D. W. (2003). Implicit memory for novel figure-ground displays includes a history of cross-border competition. Journal of Experimental Psychology: Human Perception and Performance, 29, 808-822. doi:10.1037/00961523.29.4.808

Peterson, M. A., \& Salvagio, E. (2008). Inhibitory competition in figureground perception: Context and convexity. Journal of Vision, 8(16), 4:1-13. doi:10.1167/8.16.4

Peterson, M. A., \& Skow-Grant, E. (2003). Memory and learning in figure-ground perception. In B. H. Ross \& D. Irwin (Eds.), Cognitive vision: The psychology of learning and motivation (Vol. 42, pp. 1-34). San Diego: Academic Press.

Richard, A. M., Lee, H., \& Vecera, S. P. (2008). Attentional spreading in object-based attention. Journal of Experimental Psychology: Human Perception and Performance, 34, 842-853. doi:10.1037/ 0096-1523.34.4.842

Robertson, L. C., \& Kim, M. S. (1999). Effects of perceived space on spatial attention. Psychological Science, 10, 76-79. doi:10.1111/ 1467-9280.00110

Roelfsema, P. R., Lamme, V. A. F., Spekreijse, H., \& Bosch, H. (2000). Figure-ground segregation in a recurrent network architecture. Journal of Cognitive Neuroscience, 14, 525-537. doi:10.1162/ 08989290260045756

Roller, B., Mojica, A. J., Salvagio, E., \& Peterson, M. A. (2011). Object based attention effects disappear when flanking objects are present [Abstract]. Journal of Vision, 11(11), 143. doi:10.1167 $/ 11.11 .143$

Shomstein, S. (2012). Object-based attention: Strategy vs. automaticity. WIREs Cognitive Science, 3, 163-169.
Shomstein, S., \& Yantis, S. (2002). Object-based attention: Sensory modulation or priority setting? Perception \& Psychophysics, 64, 41-51. doi:10.3758/BF03194556

Shomstein, S., \& Yantis, S. (2004). Configural and contextual prioritization in object-based attention. Psychonomic Bulletin \& Review, 11, 247-253. doi:10.3758/BF03196566

Vecera, S. P., Flevaris, A. V., \& Filapek, J. C. (2004). Exogenous spatial attention influences figure-ground assignment. Psychological Science, 15, 20-26. doi:10.1111/j.0963-7214.2004.01501004.x

Wager, E. E., Peterson, M. A., Folstein, J., \& Scalf, P. E. (2015). Groundbased inhibition: Suppressive perceptual mechanisms interact with top-down attention to reduce distractor interference. Journal of Vision, 15(8), 9:1-14. doi:10.1167/15.8.9

West, G. L., Pratt, J., \& Peterson, M. A. (2013). Attention is biased to near surfaces. Psychonomic Bulletin \& Review, 20, 1213-1220. doi:10.3758/s13423-013-0455-7

Wong, E., \& Weisstein, N. (1982). A new perceptual context-superiority effect: Line segments are more visible against a figure than against a ground. Science, 218, 587-589.

Yantis, S., \& Hillstrom, A. P. (1994). Stimulus-driven attentional capture: Evidence from equiluminant visual objects. Journal of Experimental Psychology: Human Perception and Performance, 20, 95-107. doi:10.1037/0096-1523.20.1.95

Yantis, S., \& Jonides, J. (1984). Abrupt visual onsets and selective attention: Evidence from visual search. Journal of Experimental Psychology: Human Perception and Performance, 10, 601-621. doi:10.1037/0096-1523.10.5.601

Yeari, M., \& Goldsmith, M. (2010). Is object-based attention mandatory? Strategic control over mode of attention. Journal of Experimental Psychology: Human Perception and Performance, 36, 565-579. doi:10.1037/a0016897

Yeshurun, Y., Kimchi, R., Sha'shoua, G., \& Carmel, T. (2009). Perceptual objects capture attention. Vision Research, 49, 1329-1335.

Zhao, J., Al-Aidroos, N., \& Turk-Browne, N. B. (2013). Attention is spontaneously biased toward regularities. Psychological Science, 24, 667-677. doi:10.1177/0956797612460407 